\title{
Synthesis and Antimicrobial Evaluation of Some Novel Thiazole, Pyridone, Pyrazole, Chromene, Hydrazone Derivatives Bearing a Biologically Active Sulfonamide Moiety
}

\author{
Elham S. Darwish *, Azza M. Abdel Fattah, Fawzy A. Attaby and Oqba N. Al-Shayea \\ Department of Chemistry, Faculty of Science, Cairo University, Giza 12613, Egypt; \\ E-Mails: azza682000@yahoo.com (A.M.A.F.); fattaby@hotmail.com (F.A.A.); \\ org.chemo@yahoo.com (O.N.A.-S.) \\ * Author to whom correspondence should be addressed; E-Mail: elham_darwish@yahoo.com; \\ Tel.: +20-100-588-1771; Fax: +202-570-8480.
}

Received: 10 November 2013; in revised form: 10 January 2014 / Accepted: 13 January 2014 / Published: 17 January 2014

\begin{abstract}
This study aimed for the synthesis of new heterocyclic compounds incorporating sulfamoyl moiety suitable for use as antimicrobial agents via a versatile, readily accessible $\mathrm{N}$-[4-(aminosulfonyl)phenyl]-2-cyanoacetamide (3). The 2-pyridone derivatives were obtained via reaction of cyanoacetamide with acetylacetone or arylidenes malononitrile. Cycloaddition reaction of cyanoacetamide with salicyaldehyde furnished chromene derivatives. Diazotization of $\mathbf{3}$ with the desired diazonium chloride gave the hydrazone derivatives 13a-e. Also, the reactivity of the hydrazone towards hydrazine hydrate to give Pyrazole derivatives was studied. In addition, treatment of $\mathbf{3}$ with elemental sulfur and phenyl isothiocyanate or malononitrile furnished thiazole and thiophene derivatives respectively. Reaction of $\mathbf{3}$ with phenyl isothiocyanate and $\mathrm{KOH}$ in DMF afforded the intermediate salt 17 which reacted in situ with 3-(2-bromoacetyl)-2H-chromen-2-one and methyl iodide afforded the thiazole and ketene $N, S$-acetal derivatives respectively. Finally, reaction of 3 with carbon disulfide and 1,3-dibromopropane afforded the $N$-[4-(aminosulfonyl) phenyl]-2-cyano-2-(1,3-dithian-2-ylidene)acetamide product $\mathbf{2 2}$. All newly synthesized compounds were elucidated by considering the data of both elemental and spectral analysis. The compounds were evaluated for both their in vitro antibacterial and antifungal activities and showed promising results.
\end{abstract}


Keywords: sulfamoyl; acrylamide; Pyrazole; pyridones; thiophene; thiazole; antimicrobial activity

\section{Introduction}

Cyanoacetamides and their related heterocyclic derivatives have generated a great deal of attention due to their interesting biological and therapeutic value; their pharmaceutical activities include: antimicrobial [1,2], antifungal [3], insulin releasing [4], carbonic anhydrase inhibitory [5], anti-inflammatory [6], and antitumor properties [7]. Some active sulfonamides as antibacterials are also known for their immunmodifying effects [8,9]. In addition, several thiazole derivatives possess important pharmacological activities and therefore they are useful materials in drug research. Over the past few decades, the literature has been enriched with progressive findings about the anticonvulsant activities of various substituted thiazole derivatives [10-14] that are of interest as potential neuroprotective agents [15,16]. Some 2-pyridones are also reported to possess antitumor [17], antibacterial [18] and other biological activities [19,20]. In view of these facts and as a continuation of our research program on the chemistry of butanamide [21,22], the present investigation aimed to synthesize and characterize newer hydrazones, pyridones, acrylamide, Pyrazole, thiadiazole, and thiophene incorporating sulfonamide moiety. It was found that $N$-[4-(aminosulfonyl)phenyl]2-cyanoacetamide (3) is an excellent building block for the synthesis of the target objectives.

\section{Results and Discussion}

One reason of our interest in amines is related to the conversion possibility of their $\mathrm{NH}_{2}$ group to the $\mathrm{NHCOCH}_{2} \mathrm{CN}$ group, which leads to cyanoacetamide with further useful functionalization at this position [23,24]. Cyanoacetamide $\mathbf{3}$ was synthesized by cyanoacetylation of $\mathbf{1}$ with 3,5-dimetyl-1-cyanoacetyl Pyrazole (2) [25] as previously described (Scheme 1).

Scheme 1. Synthesis of cyanoacetamide 3.

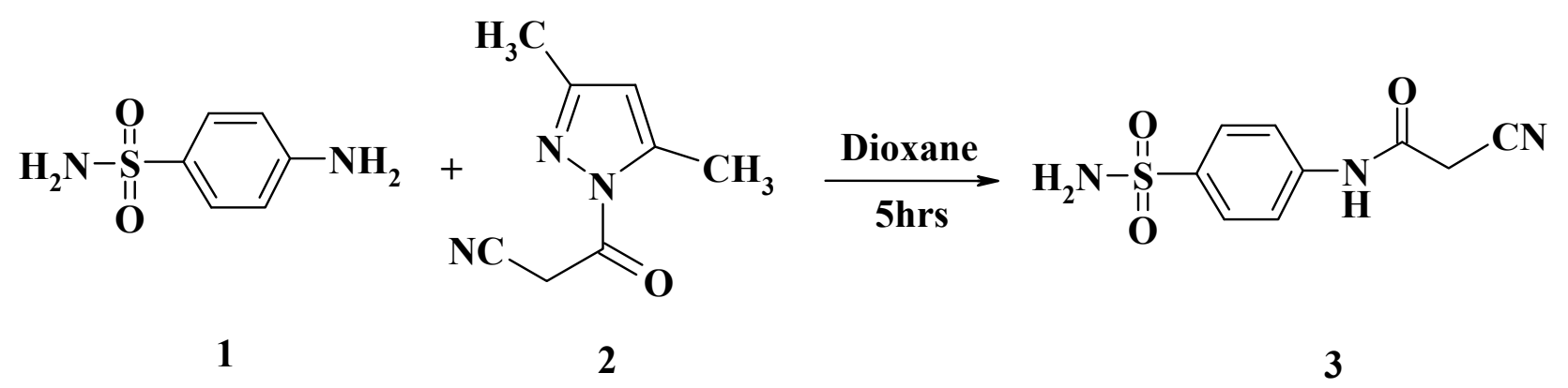

Thus, the Knoevengel condensation of the cyanoacetamide 3 with aromatic aldehydes namely benzaldehyde, $p$-anisaldehyde, and $p$-chlorobenzaldehyde furnished the corresponding arylidene derivatives 5a-c (Scheme 2). The IR spectrum of compound 5a, taken as a typical example of the series prepared, revealed absorption bands at 1680, 2220 and $3362 \mathrm{~cm}^{-1}$ corresponding to carbonyl, nitrile and $\mathrm{NH}$ functions, respectively. Its ${ }^{1} \mathrm{H}-\mathrm{NMR}$ spectrum showed signals at $\delta 8.32$ and 10.70 
( $\mathrm{D}_{2} \mathrm{O}$-exchangeable) due to $\mathrm{CH}$ and $\mathrm{NH}$ protons in addition to two aromatic protons at $\delta 7.81-7.83$. Its mass spectrum showed a molecular ion peak at $\mathrm{m} / \mathrm{z}$ 327. Pyridin-2(1H)-ones 7a-c was obtained through the reaction of the arylidene derivatives $\mathbf{5 a}-\mathbf{c}$ with malononitrile in dioxane containing piperidine as catalyst. One-pot reactions of the cyanoacetamide derivative $\mathbf{3}$ with malononitrile and the same aldehydes (1:1:1 molar ratio) at reflux temperature in the presence of piperidine afforded the 2-pyridone derivatives $7 \mathbf{a}-\mathbf{c}$. Spectroscopic data as well as elemental analyses of the obtained products were in complete agreement with the assigned structures $\mathbf{7 a - c}$. In addition, when the cyanoacetamide $\mathbf{3}$ was reacted with acetylacetone in dioxane in the presence of a catalytic amount of triethylamine, the cyclocondensation reaction occurred and the 2-pyridinone derivatives $\mathbf{1 0}$ were smoothly afforded. It can be postulated that the reaction initially proceeds via a nucleophilic attack to form the Michael adduct which in turn cyclized and eliminated two water molecules, affording the final product (Scheme 2).

Scheme 2. Synthesis of arylidene 5a-c and pyridones $7 \mathbf{a}-\mathbf{c}$ and $\mathbf{1 0 .}$

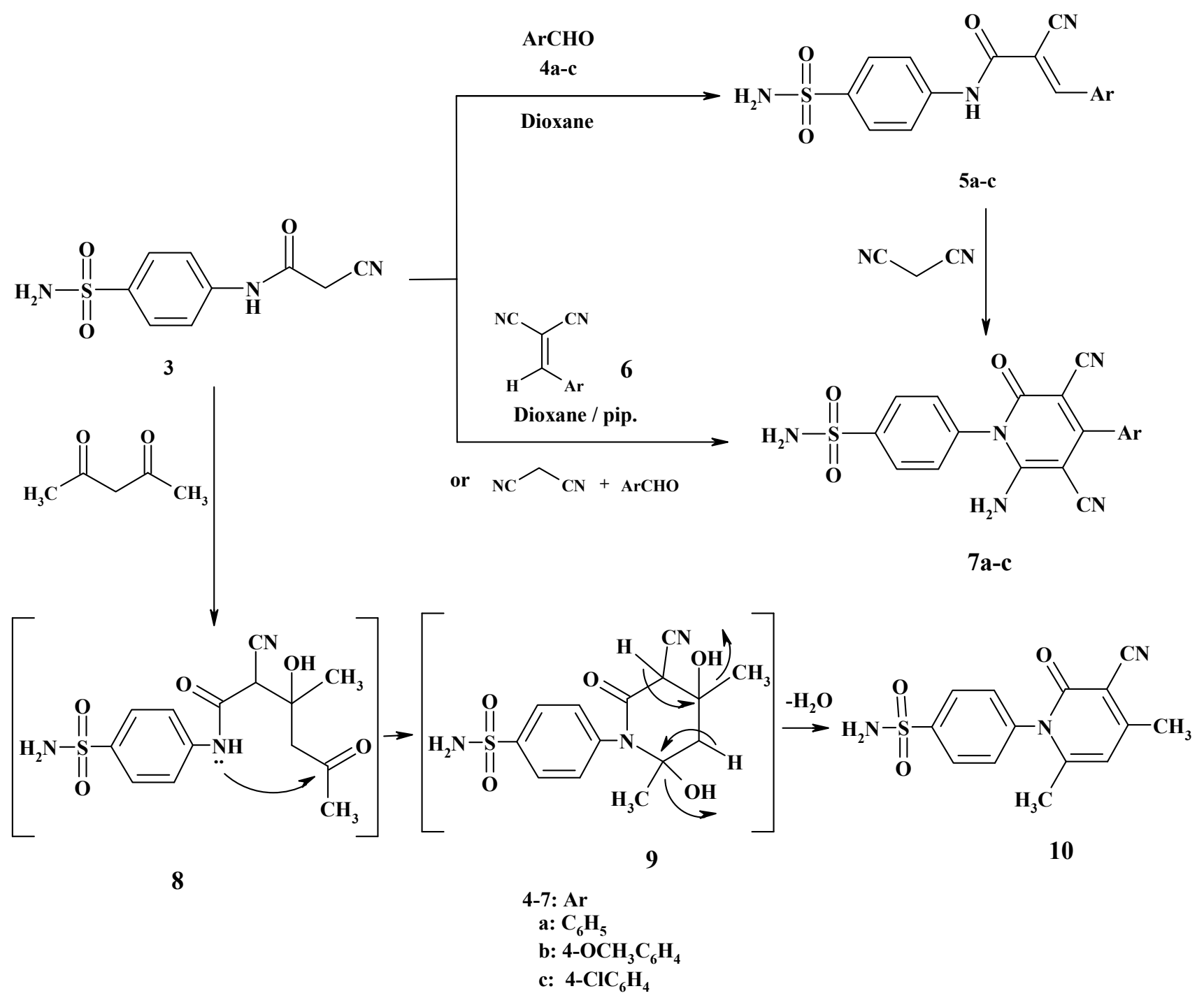

Similarly, cyclocondensation of cyanoacetamide $\mathbf{3}$ with salicyaldehyde in dioxane in the presence of a catalytic amount of piperidine afforded 2-iminochromene $\mathbf{1 1}$ in high yield. On the other hand, by interaction of 3 with salicyaldehyde in the presence of $\mathrm{AcOH} / \mathrm{AcONa}$, chromenone $\mathbf{1 2}$ was obtained in reasonably good yield. The structure of compound $\mathbf{1 2}$ was further confirmed through its synthesis upon 
hydrolysis of 11 with ethanolic $\mathrm{HCl}$. (Scheme 3). The IR spectrum of the reaction product 11 revealed the disappearance of cyano absorption band and showed absorption bands at 1680,3244 and $3318 \mathrm{~cm}^{-1}$ corresponding to carbonyl and two $\mathrm{NH}$ functions, respectively. Its ${ }^{1} \mathrm{H}-\mathrm{NMR}$ spectrum showed two $\mathrm{D}_{2} \mathrm{O}$-exchangeable signal at $\delta 9.29$ and 13.11 due to two $\mathrm{NH}$ protons, in addition to an aromatic multiplet in the region 7.58-7.83. It mass spectrum showed a molecular ion peak at $\mathrm{m} / \mathrm{z} 343$ while ${ }^{1} \mathrm{H}-\mathrm{NMR}$ spectrum of $\mathbf{1 2}$ showed one $\mathrm{D}_{2} \mathrm{O}$-exchangeable signal at $\delta 10.88$ due to one $\mathrm{NH}$ proton.

Scheme 3. Synthesis of 2-imino-2H-chromene 11 and 2-oxo- $2 H$-chromene 12.

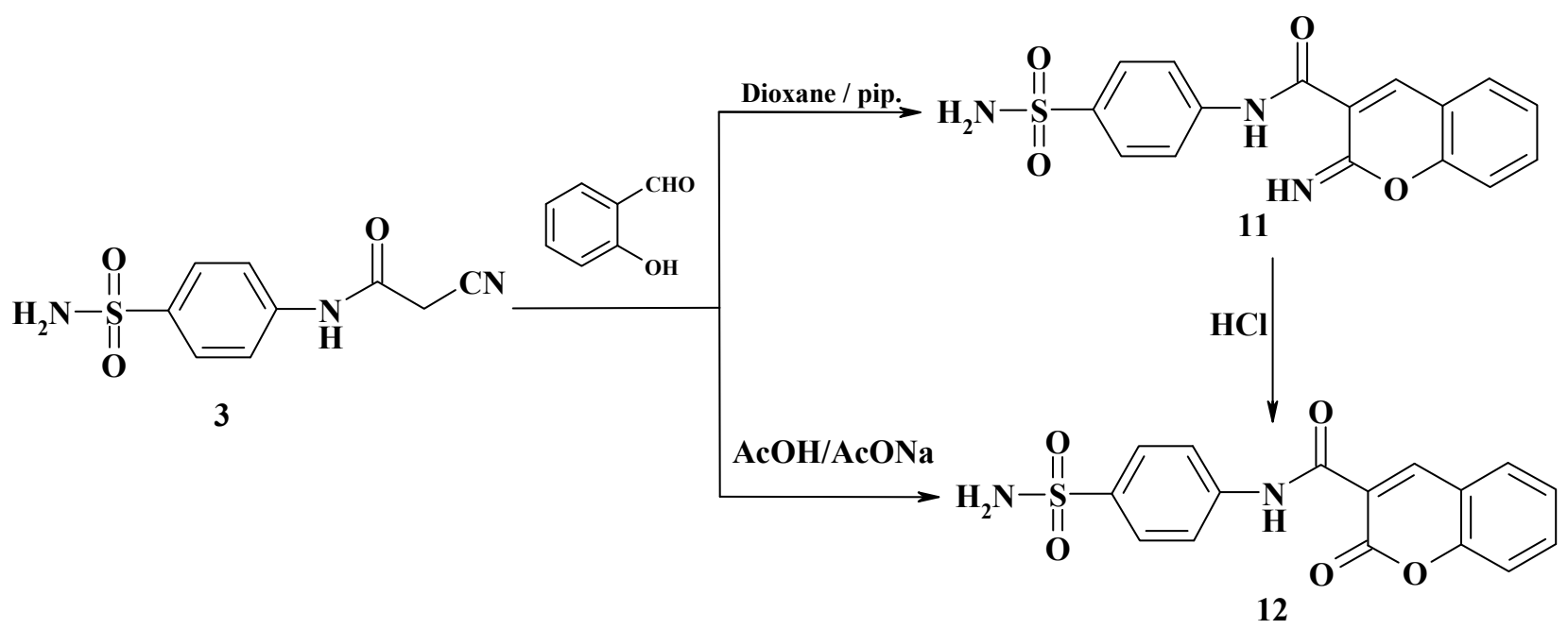

Next, we studied the reactivity of the active methylene group present in compound $\mathbf{3}$ towards diazonium salts. Thus, cyanoacetamide 3 coupled with diazonium salts, derived from the appropriate aromatic amines (4-methylaniline, 4-methoxyaniline, aniline, 4-chloroaniline, and methyl anthranilate) in pyridine to afford the respective hydrazones 13a-e (Scheme 4). Analytical and spectral data of the latter reaction products are all consistent with the proposed structures.

Scheme 4. Synthesis of hydrazones 13a-e and aminopyrazole 14a,b.<smiles>[14CH3]NN=C(C#N)C(=O)Nc1ccc(S(N)(=O)=O)cc1</smiles>

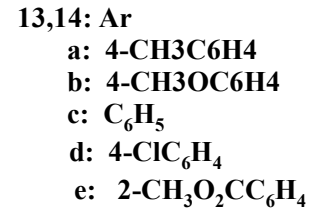


Further elucidation of the structure of $\mathbf{1 3 a}, \mathbf{b}$ came from the reaction with hydrazine hydrate to furnish the Pyrazole products $\mathbf{1 4 a}, \mathbf{b}$. The structures of compounds $\mathbf{1 4 a}, \mathbf{b}$ were confirmed based on elemental analysis and spectral data (see Experimental section).

In view of the growing biological importance of thiazole derivatives, it was considered of interest to synthesize some new derivative of thiazole. Thus, the reaction of compound $\mathbf{3}$ with phenyl isothiocyanate and elemental sulfur gave the thiazole-2-thione derivative 15. The reaction of cyanoacetamide 3 with elemental sulfur and malononitrile gave the thiophene derivative $\mathbf{1 6}$ (Scheme 5). Analytical and spectral data of the products are in agreement with the proposed structure (see Experimental section).

Scheme 5. Synthesis of aminothiazole 15 and diaminothiophene $\mathbf{1 6 .}$

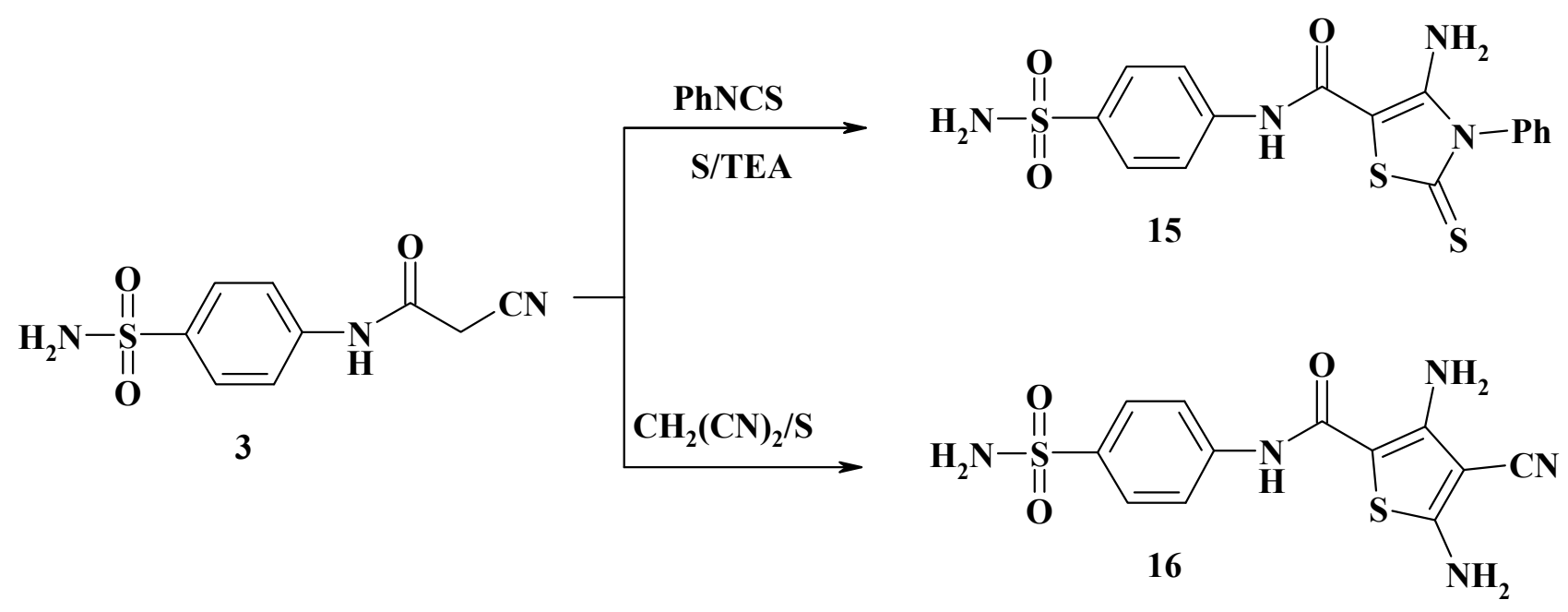

The reactivity of cyanoacetamide $\mathbf{3}$ towards isothiocyanate was investigated. Thus, when $\mathbf{3}$ was left to react with phenyl isothiocyanate in dimethylformamide, in the presence of potassium hydroxide, at room temperature, the corresponding potassium salt 17 was obtained. Heterocyclization of the intermediate 17 with chloroacetone or 3-(2-bromoacetyl)-2H-chromen-2-one furnished in each case, one isolable product (as tested by TLC). Probably the reaction proceeds via nucleophilic displacement of the halogen atom to give an $S$-alkylated intermediate followed by loss of water of the latter intermediate to give thiazole derivatives 18 and 19 as the final products. The structures of the products 18 and 19 as well as the rejection of $\mathbf{1 8}$ ' were determined from spectroscopic and elemental analytical data. Analytical and spectral data of the product are in agreement with the proposed structure (see Experimental section). Furthermore, the non-isolated potassium salt was methylated by treatment with methyl iodide to afford the novel ketene $N, S$-acetal 20 (Scheme 6). The structure of the synthesized product was established on the basis of their elemental analysis and spectral data (see Experimental section).

Stirring the cyanoacetamide 3 with carbon disulfide in the presence of potassium hydroxide in $N, N$-dimethylformamide followed by cycloalkylation with 1,3-dibromopropane afforded $N$-[4-(amino-sulfonyl)phenyl]-2-cyano-2-(1,3-dithian-2-ylidene)acetamide (22) (Scheme 7). The IR spectra of compound 22 showed characteristic bands for $\mathrm{NH}, \mathrm{CH}-$ aliphatic, $\mathrm{C} \equiv \mathrm{N}$ and $\mathrm{C}=\mathrm{O}$ groups. ${ }^{1} \mathrm{H}-\mathrm{NMR}$ spectrum of compound 22 showed signals for dithiene moiety at $\delta 2.18 \mathrm{ppm}(\mathrm{m}, 2 \mathrm{H}$, $\left.J=6.80 \mathrm{~Hz}, \mathrm{CH}_{2}\right), 3.04\left(\mathrm{t}, 2 \mathrm{H}, J=6.60 \mathrm{~Hz}, \mathrm{CH}_{2}\right), 3.19$ (t, $2 \mathrm{H}, J=6.60 \mathrm{~Hz}, \mathrm{CH}_{2}$ ). Mass spectrum of 22 showed a molecular ion peak at $m / z: 355$ with a base peak at $m / z: 183(100 \%)$. 
Scheme 6. Synthesis of thiazoles 18, 19 and acrylamide 20.

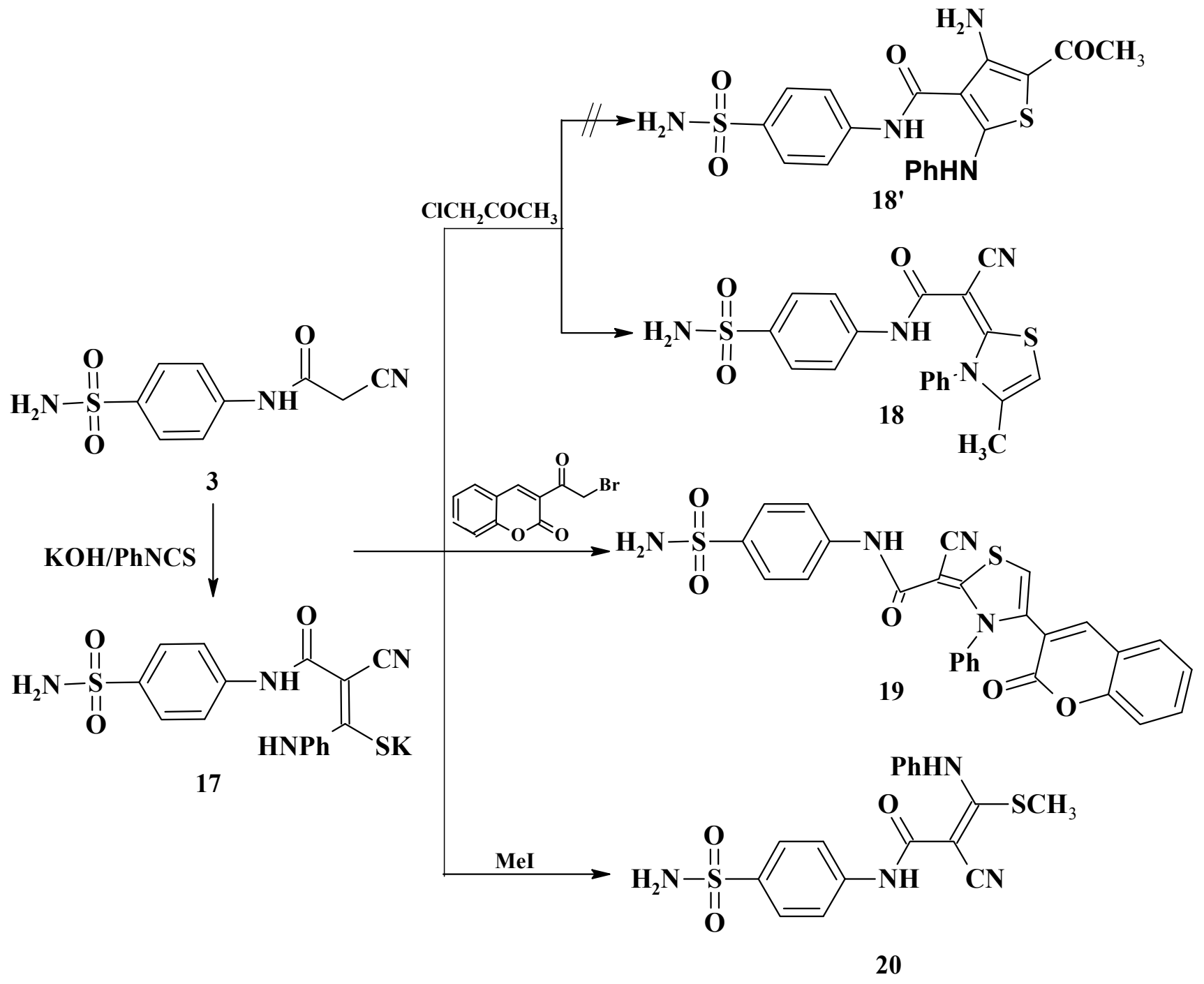

Scheme 7. Synthesis of 1,3-dithian-2-ylidene 22.<smiles>N#CCC(=O)Nc1ccc(S(N)(=O)=O)cc1</smiles>

3<smiles>N#CC(C(=O)Nc1ccc(S(N)(=O)=O)cc1)=C1SCCCS1</smiles><smiles>CC(C(=O)Nc1ccc(S(N)(=O)=O)cc1)=C([Si])[Si]</smiles>

21 $\mathrm{BrCH}_{2} \mathrm{CH}_{2} \mathrm{CH}_{2} \mathrm{Br}$ 


\subsection{Screening for Antimicrobial Activity}

The newly synthesized compounds $5 \mathbf{a}, 5 \mathbf{c}, 7 \mathbf{b}, 7 \mathbf{c}, 10,11,13 \mathbf{b}, 14 \mathbf{a}, 15,16,18,19$ and 22 were evaluated for their in vitro antibacterial activity against Streptococcus pneumoniae (RCMB-010010) $(S P)$ and Bacillis subtilis (RCMB-010067) (BS) as examples of Gram-positive bacteria and Pseudomonas aeruginosa (RCMB-010043) (PA) and Escherichia coli (RCMB-010052) (EC) as examples of Gram-negative bacteria. They were also evaluated for their in vitro antifungal activity against Aspergillus fumigatus (RCMB-02568) (AF), Syncephalastrum racemosum (RCMB-05922) (SR), Geotricum candidum (RCMB-05097) (GC) and Candida albicans (RCMB-05036) (CA) fungal strains. Inhibition zone diameter (IZD) in $\mathrm{mm}$ was used as criterion for the antimicrobial activity using the diffusion technique [26-28]. The fungicide Amphotericin B and the bactericides Ampicillin and Gentamicin were used as references to evaluate the potency of the tested compounds under the same conditions. The results are depicted in Table 1. As seen from the data present in Table 1, Streptococcus pneumoniae and Bacillis subtilis are sensitive to all tested compounds except compounds 11 and 16; furthermore, Pseudomonas aeruginosa is sensitive to compounds 5a, 7c and 15, while Escherichia coli is sensitive to $5 \mathbf{a}, \mathbf{7 b}, \mathbf{7 c}, \mathbf{1 0}, \mathbf{1 3 b}, \mathbf{1 4 a}, \mathbf{1 5}, \mathbf{1 8}, 19$ and 22 except compounds 11 and 16. All tested compounds except compound $\mathbf{1 6}$ exhibit antifungal activity against the three tested fungi species Aspergillus fumigatus, Syncephalastrum racemosum and Geotricum candidum. Also the Candida albicans strain is sensitive to compounds $\mathbf{5 a}, \mathbf{5 c}, \mathbf{7 c}, \mathbf{1 0}, \mathbf{1 1}$. The high activity of $5 \mathbf{a}, 7 \mathbf{c}$ and 10 is attributed to the presence of pharmacological active arylidene moiety in compound $\mathbf{5 a}$ and pyridone ring in $\mathbf{7 c}$ and $\mathbf{1 0}$. The inactivity of compound $\mathbf{1 6}$ against the tested bacteria and fungi is due to the presence of a thiophene ring.

Table 1. Antibacterial and antifungal activities of the synthesized compounds $(\mathbf{5 a}, \mathbf{5 c}, \mathbf{7 b}$, $7 c, 10,11,13 b, 14 a, 15,16,18,19$ and 22).

\begin{tabular}{|c|c|c|c|c|c|c|c|c|}
\hline \multirow{4}{*}{$\begin{array}{c}\text { Comp. } \\
\text { Standard } \\
\text { values } \\
\end{array}$} & \multicolumn{8}{|c|}{ Inhibition zone diameter $(\mathrm{cm})$} \\
\hline & \multicolumn{2}{|c|}{ Gram (+) } & \multicolumn{2}{|c|}{$\operatorname{Gram}(-)$} & \multicolumn{4}{|c|}{ Fungi } \\
\hline & $(S P)$ & $(B S)$ & $(\boldsymbol{P A})$ & $(E C)$ & $(A F)$ & $(S R)$ & $(G C)$ & $(C A)$ \\
\hline & $23.8 \pm 0.2$ & $32.4 \pm 0.3$ & $17.3 \pm 0.1$ & $19.9 \pm 0.3$ & $23.7 \pm 0.2$ & $19.7 \pm 0.2$ & $28.7 \pm 0.2$ & $25.4 \pm 0.1$ \\
\hline $5 \mathbf{a}$ & $18.9 \pm 0.44$ & $21.7 \pm 0.25$ & $11.6 \pm 0.19$ & $15.4 \pm 0.39$ & $20.2 \pm 0.55$ & $16.3 \pm 0.25$ & $22.4 \pm 0.58$ & $19.6 \pm 0.33$ \\
\hline $5 \mathrm{c}$ & $16.3 \pm 0.55$ & $18.3 \pm 0.25$ & NA & NA & $17.3 \pm 0.44$ & $12.6 \pm 0.25$ & $19.0 \pm 0.58$ & $16.9 \pm 0.25$ \\
\hline $7 \mathbf{b}$ & $16.9 \pm 0.58$ & $18.2 \pm 0.44$ & NA & $11.9 \pm 0.63$ & $15.7 \pm 0.33$ & $13.8 \pm 0.25$ & $18.3 \pm 0.34$ & NA \\
\hline $7 \mathrm{c}$ & $18.3 \pm 0.25$ & $22.6 \pm 0.44$ & $13.1 \pm 0.32$ & $20.3 \pm 0.09$ & $20.6 \pm 0.58$ & $16.7 \pm 0.33$ & $22.4 \pm 0.36$ & $17.6 \pm 0.58$ \\
\hline 10 & $16.7 \pm 0.36$ & $19.2 \pm 0.27$ & NA & $13.6 \pm 0.36$ & $16.8 \pm 0.39$ & $13.4 \pm 0.58$ & $19.6 \pm 0.19$ & $15.9 \pm 0.44$ \\
\hline 11 & NA & NA & NA & NA & $15.7 \pm 0.36$ & $11.2 \pm 0.33$ & $17.3 \pm 0.44$ & $13.3 \pm 0.36$ \\
\hline $13 b$ & $12.3 \pm 0.58$ & $12.7 \pm 0.37$ & NA & $8.5 \pm 0.37$ & $17.6 \pm 0.58$ & $15.4 \pm 0.25$ & $12.6 \pm 0.38$ & NA \\
\hline $14 a$ & $17.5 \pm 0.44$ & $19.8 \pm 0.63$ & NA & $18.9 \pm 0.25$ & $15.3 \pm 0.55$ & $13.4 \pm 0.35$ & $11.5 \pm 0.58$ & NA \\
\hline 15 & $15.0 \pm 0.43$ & $17.4 \pm 0.53$ & $12.3 \pm 0.25$ & $17.8 \pm 0.03$ & $11.3 \pm 0.34$ & $12.1 \pm 0.25$ & $15.3 \pm 0.38$ & NA \\
\hline 16 & NA & NA & NA & NA & NA & NA & NA & NA \\
\hline 18 & $16.9 \pm 0.58$ & $18.2 \pm 0.44$ & NA & $11.9 \pm 0.63$ & $16.2 \pm 0.36$ & $15.0 \pm 0.44$ & $17.6 \pm 0.58$ & NA \\
\hline 19 & $12.9 \pm 0.63$ & $13.2 \pm 0.58$ & NA & $10.8 \pm 0.44$ & $18.7 \pm 0.36$ & $16.9 \pm 0.27$ & $13.4 \pm 0.65$ & NA \\
\hline 22 & $12.3 \pm 0.58$ & $12.7 \pm 0.37$ & NA & $10.8 \pm 0.44$ & $17.6 \pm 0.58$ & $15.4 \pm 0.25$ & $12.6 \pm 0.38$ & NA \\
\hline
\end{tabular}

Data are expressed in the form of mean \pm SD. Mean zone of inhibition in $\mathrm{mm} \pm$ standard deviation beyond well diameter; ( $6 \mathrm{~mm})$ produced on a range of environmental and clinically pathogenic microorganism using $(5 \mathrm{mg} / \mathrm{mL})$ concentration of tested sample (100 $\mu \mathrm{L}$ was tested). 


\section{Experimental Section}

\subsection{General Experimental Procedures}

All melting points were measured on an Electrothermal Gallenkamp apparatus (Weiss-Gallenkamp, London, UK). The infrared spectra were recorded in potassium bromide discs on a Pye Unicam SP3300 and Shimadzo FT IR 8101 PC infrared spectrophotometers (Pye Unicam Ltd., Cambridge, UK and Shimadzu, Tokyo, Japan, respectively). The ${ }^{1} \mathrm{H}-\mathrm{NMR}$ spectra were recorded on a Varian Mercury VXR-300 spectrometer (300 MHz, Vernon Hills, IL, USA). The mass spectra were recorded on a GCMS-Q1000-EX Shimadzu and GCMS 5988-A HP spectrometers (Kyoto, Japan), the ionizing voltage was $70 \mathrm{eV}$. Elemental analyses were carried out at the Micro-analytical Center of Cairo University, Giza, Egypt. The biological evaluation of the products was carried out in the Medical Mycology Laboratory of the Regional Center for Mycology and Biotechnology of Al-Azhar University, Cairo, Egypt. The starting material 3-(2-bromoacetyl)-2H-chromen-2-one was prepared as previously reported in the literature $[29,30]$.

\subsection{Synthetic Procedures}

\subsubsection{2-Cyano- $N$-(4-sulfamoylphenyl)acetamide (3)}

A mixture of 1 (3.44 g, $20 \mathrm{mmol}$ ) and 3,5-dimetyl-1-cyanoacetyl Pyrazole (2) (3.26 g, $20 \mathrm{mmol})$ in dioxane $(20 \mathrm{~mL})$ was refluxed for $3 \mathrm{~h}$. The reaction mixture was poured into crushed ice. The resulting precipitate was filtrated off, dried, and crystallized from $\mathrm{DMF} / \mathrm{MeOH}(1: 3)$ to give 3; Yield (70\%), mp $248{ }^{\circ} \mathrm{C}$ (from DMF/MeOH) (lit. mp $230{ }^{\circ} \mathrm{C}$ ) [31]; IR (KBr) v $v_{\max }: 3337,3236,3102\left(\mathrm{NH}, \mathrm{NH}_{2}\right), 2269$ $(\mathrm{C} \equiv \mathrm{N}), 1687(\mathrm{C}=\mathrm{O}) \mathrm{cm}^{-1} ;{ }^{1} \mathrm{H}-\mathrm{NMR}\left(\mathrm{DMSO}-d_{6}\right): \delta 3.96\left(\mathrm{~s}, 2 \mathrm{H}, \mathrm{CH}_{2}\right), 7.29\left(\mathrm{~s}, 2 \mathrm{H}, \mathrm{D}_{2} \mathrm{O}\right.$-exchangeable $\left.\mathrm{NH}_{2}\right), 7.77(\mathrm{~d}, 2 \mathrm{H}, J=9 \mathrm{~Hz}), 7.80(\mathrm{~d}, 2 \mathrm{H}, J=9 \mathrm{~Hz}), 10.63\left(\mathrm{~s}, 1 \mathrm{H}, \mathrm{D}_{2} \mathrm{O}\right.$-exchangeable $\left.\mathrm{NH}\right) . \mathrm{MS} m / z(\%)$ : $241\left(\mathrm{M}^{+}+2,0.7\right), 240\left(\mathrm{M}^{+}+1,5.8\right), 239\left(\mathrm{M}^{+}, 12.7\right), 238$ (100), 222 (34.9), 172 (25.3), 159 (29.7), 132 (19.1), 108 (47.3), 92 (46.3), 90 (25.1), 75 (15.9). Anal. Calcd for $\mathrm{C}_{9} \mathrm{H}_{9} \mathrm{~N}_{3} \mathrm{O}_{3} \mathrm{~S}$ (239.25): C, 45.18; H, 3.79; N, 17.56; S, 13.40. Found: C, 45.11; H, 3.70; 65, N, 17.42; S, 13.30\%.

\subsection{2. $N$-[4-(Aminosulfonyl)phenyl]-3-aryl-2-cyanoacrylamide (5a-c)}

General Procedure: To a solution of cyanoacetanilide $3(0.239 \mathrm{~g}, 1 \mathrm{mmol})$ and the appropriate aromatic aldhyeds $(1 \mathrm{mmol})$ in dioxane $(20 \mathrm{~mL})$, was added few drops of piperidine and the reaction mixture was refluxed for $6 \mathrm{~h}$. The solid product so formed was filtered off, washed with EtOH and then recrystallized from proper solvent to give $\mathbf{5 a}-\mathbf{c}$.

\subsubsection{2-Cyano-3-phenyl- $N$-(4-sulfamoylphenyl)prop-2-enamide (5a)}

Yield (45\%), mp $322{ }^{\circ} \mathrm{C}$ (from dioxane/ethanol); IR (KBr) $v_{\max }$ : 3362, 3316, $3262\left(\mathrm{NH}, \mathrm{NH}_{2}\right)$, $2220(\mathrm{C} \equiv \mathrm{N}), 1680(\mathrm{C}=\mathrm{O}) \mathrm{cm}^{-1} ;{ }^{1} \mathrm{H}-\mathrm{NMR}\left(\mathrm{DMSO}-d_{6}\right): \delta 7.29\left(\mathrm{~s}, 2 \mathrm{H}, \mathrm{D}_{2} \mathrm{O}\right.$-exchangeable $\left.\mathrm{NH}_{2}\right)$, $7.63(\mathrm{~d}, 2 \mathrm{H}, J=9 \mathrm{~Hz}), 7.80(\mathrm{~d}, 2 \mathrm{H}, J=9 \mathrm{~Hz}), 7.81-7.83$ (m, 5H, ArH), 8.32 (s, 1H, olefinicH), 10.70 (s, 1H, $\mathrm{D}_{2} \mathrm{O}$-exchangeable NH). MS m/z (\%): 327 ( $\left.\mathrm{M}^{+}, 18.2\right), 128$ (40.4), 104 (10.7), 156 (100), 
77 (23.9); Anal. Calcd for $\mathrm{C}_{16} \mathrm{H}_{13} \mathrm{~N}_{3} \mathrm{O}_{3} \mathrm{~S}$ (327.35): C, 58.70; H, 4.00; N, 12.84; S, 9.80. Found: C, 58.65; $\mathrm{H}, 3.88 ; \mathrm{N}, 12.72 ; \mathrm{S}, 9.77 \%$.

\subsubsection{2-Cyano-3-(4-methoxyphenyl)- $N$-(4-sulfamoylphenyl)prop-2-enamide (5b)}

Yield (90\%), mp $292{ }^{\circ} \mathrm{C}$ (from dioxane/ethanol); IR (KBr) $v_{\max }: 3312,3266,3106\left(\mathrm{NH}, \mathrm{NH}_{2}\right)$, $2218(\mathrm{C} \equiv \mathrm{N}), 1682(\mathrm{C}=\mathrm{O}) \mathrm{cm}^{-1} ;{ }^{1} \mathrm{H}-\mathrm{NMR}\left(\mathrm{DMSO}-d_{6}\right): \delta 3.87\left(\mathrm{~s}, 3 \mathrm{H}, \mathrm{OCH}_{3}\right), 7.19(\mathrm{~d}, 2 \mathrm{H}, J=9 \mathrm{~Hz}), 7.27$ (s, 2H, $\mathrm{D}_{2} \mathrm{O}$-exchangeable $\left.\mathrm{NH}_{2}\right), 7.82-7.83(\mathrm{~m}, 4 \mathrm{H}, \mathrm{ArH}), 8.04(\mathrm{~d}, 2 \mathrm{H}, J=9 \mathrm{~Hz}), 8.24(\mathrm{~s}, 1 \mathrm{H}$, olefinicH), 10.56 (s, 1H, $\mathrm{D}_{2} \mathrm{O}$-exchangeable $\left.\mathrm{NH}\right)$; $\mathrm{MS} m / z$ (\%): 357 ( $\left.\mathrm{M}^{+}, 11.3\right), 199$ (0.3), 187 (12.6), 186 (100), 158 (19.2), 77 (7.6); Anal. Calcd for C17H15N3O4S (357.38): C, 57.13; H, 4.23; N, 11.76; S, 8.97. Found: C, 57.00; H, 4.18; N, 11.55; S, 8.67\%.

\subsubsection{2-Cyano-3-(4-chlorophenyl)- $N$-(4-sulfamoylphenyl)prop-2-enamide (5c)}

Yield (63\%), mp $286{ }^{\circ} \mathrm{C}$ (from dioxane/ethanol); IR (KBr) $v_{\max }: 3388,3329,3262\left(\mathrm{NH}, \mathrm{NH}_{2}\right), 2218$ $(\mathrm{C} \equiv \mathrm{N}), 1688(\mathrm{C}=\mathrm{O}) \mathrm{cm}^{-1}{ }^{1} \mathrm{H}-\mathrm{NMR}\left(\mathrm{DMSO}-d_{6}\right): \delta 7.37\left(\mathrm{~s}, 2 \mathrm{H}, \mathrm{D}_{2} \mathrm{O}\right.$-exchangeable $\left.\mathrm{NH}_{2}\right), 7.76(\mathrm{~d}, 2 \mathrm{H}$, $J=9 \mathrm{~Hz}), 7.91-8.07$ (m, 4H, ArH), 8.10 (d, 2H, $J=9 \mathrm{~Hz}), 8.39$ (s, 1H, olefinicH), $10.78(\mathrm{~s}, 1 \mathrm{H}$, $\mathrm{D}_{2} \mathrm{O}$-exchangeable NH); MS m/z (\%): $361\left(\mathrm{M}^{+}, 19.4\right), 191$ (17.3), 190 (100), 162 (27.9), 127 (29.6), 123 (4.6), 111 (5.8), 99 (5.2), 75 (13.8); Anal. Calcd for $\mathrm{C}_{16} \mathrm{H}_{12} \mathrm{ClN}_{3} \mathrm{O}_{3} \mathrm{~S}$ (361.80): C, 53.11; H, 3.34; Cl, 9.80; N, 11.61; S, 8.86. Found: C, 53.01; H, 3.29; Cl, 9.72; N, 11.51; S, 8.76\%.

\subsubsection{Synthesis of Pyridines 7a-c}

Method A: A mixture of 5 (10 mmol) and malononitrile $(0.66 \mathrm{~g}, 10 \mathrm{mmol})$ in ethanol $(30 \mathrm{~mL})$ containing piperidine $(0.5 \mathrm{~mL})$ was heated under reflux for $3 \mathrm{~h}$. After cooling, the precipitate was filtered off, washed with ethanol and then recrystallized from the proper solvent to give $7 \mathbf{a}-\mathbf{c}$.

Method B: Equimolar amounts of $3(10 \mathrm{mmol})$ and the appropriate 2-(arylidene)-malononitrile (namely 2-(benzylidene)-malononitrile, 2-(4-methoxybenzylidene)-malononitrile, and 2-(4-chloro benzylidene)-malononitrile $)(10 \mathrm{mmol})$ in ethanol $(30 \mathrm{~mL})$ was treated with piperidine $(0.5 \mathrm{~mL})$ and the reaction mixture was heated under reflux for $3 \mathrm{~h}$. After cooling, the precipitate was filtered off, washed with ethanol and then recrystallized from the proper solvent to give $7 \mathbf{a}-\mathbf{c}$.

Method C: A mixture of $\mathbf{3}(10 \mathrm{mmol})$, the appropriate aldehyde (namely benzaldehyde, $p$-anisaldehyde, and $p$-chlorobenzaldehyde) $(10 \mathrm{mmol})$, piperidine $(10 \mathrm{mmol})$, and malononitrile $(0.66 \mathrm{~g}, 10 \mathrm{mmol})$ in ethanol $(30 \mathrm{~mL})$ was heated under reflux for $3 \mathrm{~h}$. After cooling, the precipitate was filtered off, washed with ethanol and then recrystallized from the proper solvent to give $7 \mathbf{a}-\mathbf{c}$.

3.2.7. 4-(6-Amino-3,5-dicyano-2-oxo-4-phenylpyridin-1(2H)-yl)benzenesulfonamide (7a)

Yield (54\%), mp $316{ }^{\circ} \mathrm{C}$ (from dioxane/ethanol); IR (KBr) $v_{\max }: 3458,3332,3212\left(\mathrm{NH}, \mathrm{NH}_{2}\right), 2216$ $(\mathrm{C} \equiv \mathrm{N}), 1672(\mathrm{C}=\mathrm{O}) \mathrm{cm}^{-1} ;{ }^{1} \mathrm{H}-\mathrm{NMR}\left(\mathrm{DMSO}-d_{6}\right): \delta 7.50\left(\mathrm{~s}, 2 \mathrm{H}, \mathrm{D}_{2} \mathrm{O}\right.$-exchangeable $\left.\mathrm{NH}_{2}\right), 7.51-7.58$ (m, 7H, ArH, $\left.\mathrm{NH}_{2}\right), 7.60$ (d, 2H, $\left.J=9 \mathrm{~Hz}\right), 8.01$ (d, 2H, $\left.J=9 \mathrm{~Hz}\right)$; MS $m / z(\%): 392\left(\mathrm{M}^{+}+1,10.7\right), 391$ $\left(\mathrm{M}^{+}, 7.8\right), 237$ (7.8), 155 (9.2), 129 (8.7), 81 (46.5), 69 (100). Anal. Calcd for $\mathrm{C}_{19} \mathrm{H}_{13} \mathrm{~N}_{5} \mathrm{O}_{3} \mathrm{~S}(391.40)$ : C, 58.30; H, 3.35; N, 17.89; S, 8.19. Found: C, 58.20; H, 3.34; N, 17.86; S, 8.15\%. 
3.2.8. 4-[6-Amino-3,5-dicyano-4-(4-methoxyphenyl)-2-oxopyridin-1(2H)-yl]benzenesulfonamide (7b)

Yield (56\%), mp $350{ }^{\circ} \mathrm{C}$ (from dioxane/ethanol); IR (KBr) $v_{\max }: 3311,3208,3078\left(\mathrm{NH}, \mathrm{NH}_{2}\right), 2214$ $(\mathrm{C} \equiv \mathrm{N}), 1659(\mathrm{C}=\mathrm{O}) \mathrm{cm}^{-1}$; ${ }^{1} \mathrm{H}-\mathrm{NMR}\left(\mathrm{DMSO}-d_{6}\right): \delta 3.88\left(\mathrm{~s}, 3 \mathrm{H}, \mathrm{OCH}_{3}\right), 7.12\left(\mathrm{~s}, 2 \mathrm{H}, \mathrm{D}_{2} \mathrm{O}\right.$-exchangeable $\left.\mathrm{NH}_{2}\right), 7.50-7.52\left(\mathrm{~m}, 6 \mathrm{H}, \mathrm{ArH}, \mathrm{NH}_{2}\right), 7.62(\mathrm{~d}, 2 \mathrm{H}, J=9 \mathrm{~Hz}), 8.00(\mathrm{~d}, 2 \mathrm{H}, J=9 \mathrm{~Hz}),{ }^{13} \mathrm{C}-\mathrm{NMR}: \delta 55.3$, $66.3,75.4,87.8,114.0,116.5,120.1,126.5,127.7,129.7,132.7,136.8,145.3,156.9,159.5,161.1$; MS m/z (\%): $422\left(\mathrm{M}^{+}+1,15.1\right), 421\left(\mathrm{M}^{+}, 3.9\right), 336$ (11.2), 265 (12.9), 229 (15.1), 195 (13.6), 185 (34.8), 157 (22.6), 82 (34.4), 55.1 (100). Anal. Calcd for $\mathrm{C}_{20} \mathrm{H}_{15} \mathrm{~N}_{5} \mathrm{O}_{4} \mathrm{~S}$ (421.43): C, 57.00; H, 3.59; N, 16.62; S, 7.61. Found: C, 56.98; H, 3.53; N, 16.55; S, 7.58\%.

\subsubsection{4-[6-Amino-4-(4-chlorophenyl)-3,5-dicyano-2-oxopyridin-1(2H)-yl]benzenesulfonamide (7c)}

Yield (60\%), mp $>300^{\circ} \mathrm{C}$ (from dioxane/ethanol); IR (KBr) $v_{\max }: 3348,3208,3097\left(\mathrm{NH}, \mathrm{NH}_{2}\right), 2219$ $(\mathrm{C} \equiv \mathrm{N}), 1661(\mathrm{C}=\mathrm{O}) \mathrm{cm}^{-1} ;{ }^{1} \mathrm{H}-\mathrm{NMR}\left(\mathrm{DMSO}-d_{6}\right): \delta 7.51\left(\mathrm{~s}, 2 \mathrm{H}, \mathrm{D}_{2} \mathrm{O}\right.$-exchangeable $\left.\mathrm{NH}_{2}\right), 7.56-7.59$ (m, 4H, ArH), 7.69 (d, 2H, $J=9 \mathrm{~Hz}), 8.01$ (d, 2H, $J=9 \mathrm{~Hz}), 8.10$ (s, 2H, $\mathrm{D}_{2} \mathrm{O}$-exchangeable $\mathrm{NH}_{2}$ ); MS $m / z(\%): 427\left(\mathrm{M}^{+}+2,46.3\right), 426\left(\mathrm{M}^{+}+1,46.0\right), 425\left(\mathrm{M}^{+}, 100\right), 397$ (38.0), 313 (22.4), 269 (18.4), 156 (25.8), 132 (28.2), 111 (27.3), 106 (22.4), 91 (41.4), 80 (29.1), 77 (55.2). Anal. Calcd for $\mathrm{C}_{19} \mathrm{H}_{12} \mathrm{ClN}_{5} \mathrm{O}_{3} \mathrm{~S}$ (425.84): C, 53.59; H, 2.84; Cl, 8.33; N, 16.45; S, 7.53. Found: C, 53.46; H, 2.80; $\mathrm{Cl}, 8.30 ; \mathrm{N}, 16.42 ; \mathrm{S}, 7.50 \%$.

3.2.10. 4-(3-Cyano-4,6-dimethyl-2-oxopyridin-1(2H)-yl)benzenesulfonamide (10)

To a mixture of cyanoacetanilide $3(1.20 \mathrm{~g}, 5 \mathrm{mmol})$ and acetylacetone $(0.50 \mathrm{~g}, 1 \mathrm{mmol})$ in dioxane $(20 \mathrm{~mL})$, triethylamine $(0.5 \mathrm{~mL})$ was added and the reaction mixture was refluxed for $8 \mathrm{~h}$. On cooling, the separated solid was filtered, washed with ethanol and crystallized from DMF to afford the corresponding 4-(3-cyano-4,6-dimethyl-2-oxopyridin-1(2H)-yl)benzenesulfonamide (10). Yield (64\%), $\mathrm{mp}>300^{\circ} \mathrm{C}$ (from DMF); IR (KBr) $v_{\max }: 3314,3174,3083\left(\mathrm{NH}, \mathrm{NH}_{2}\right), 2223(\mathrm{C} \equiv \mathrm{N}), 1648(\mathrm{C}=\mathrm{O}) \mathrm{cm}^{-1}$; ${ }^{1} \mathrm{H}-\mathrm{NMR}$ (DMSO- $\left.d_{6}\right): \delta 1.98\left(\mathrm{~s}, 3 \mathrm{H}, \mathrm{CH}_{3}\right), 2.40\left(\mathrm{~s}, 3 \mathrm{H}, \mathrm{CH}_{3}\right), 6.49(\mathrm{~s}, 1 \mathrm{H}$, pyridineH), 7.52-7.57 (m, 4H, ArH, $\left.\mathrm{NH}_{2}\right), 7.99$ (d, 2H, $\left.J=9 \mathrm{~Hz}, \mathrm{ArH}\right) ; \mathrm{MS} m / z$ (\%): 303 (M+ 51.9), 302 (100), 274 (10.6), 223 (21.5), 171 (0.9), 156 (2.8), 78 (23.6), 50 (20.6). Anal. Calcd for $\mathrm{C}_{14} \mathrm{H}_{13} \mathrm{~N}_{3} \mathrm{O}_{3} \mathrm{~S}$ (303.33): C, 55.43; H, 4.32; N, 13.85; S, 10.57. Found: C, 55.33; H, 4.28; N, 13.78; S, 10.52\%.

\subsubsection{2-Imino- $N$-(4-sulfamoylphenyl)-2H-chromene-3-carboxamide (11)}

A mixture of equimolar amounts of $3(1.20 \mathrm{~g}, 5 \mathrm{mmol})$ and salicyaldehyde $(0.61 \mathrm{~g}, 5 \mathrm{mmol})$ in 1,4-dioxane $(25 \mathrm{~mL})$ containing a catalytic amount of piperidine was heated under reflux for $2 \mathrm{~h}$. The solid product formed was collected by filtration and recrystallized from dioxane/ethanol $(3: 1)$ to give 11. Yield (90\%), mp $294{ }^{\circ} \mathrm{C}$ (from dioxane/ethanol); IR (KBr) $v_{\max }$ : 3318, $3244\left(\mathrm{NH}, \mathrm{NH}_{2}\right)$, $1680(\mathrm{C}=\mathrm{O}) \mathrm{cm}^{-1} ;{ }^{1} \mathrm{H}-\mathrm{NMR}\left(\mathrm{DMSO}-d_{6}\right): \delta 7.25\left(\mathrm{~s}, 2 \mathrm{H}, \mathrm{D}_{2} \mathrm{O}\right.$-exchangeable $\left.\mathrm{NH}_{2}\right), 7.32(\mathrm{~d}, 2 \mathrm{H}, J=9 \mathrm{~Hz}$, $\operatorname{ArH}), 7.58-7.63(\mathrm{~m}, 4 \mathrm{H}, \operatorname{ArH}), 7.83(\mathrm{~d}, 2 \mathrm{H}, J=9 \mathrm{~Hz}, \mathrm{ArH}), 8.58(\mathrm{~s}, 1 \mathrm{H}, \mathrm{CH}), 9.29(\mathrm{~s}, 1 \mathrm{H}$, $\mathrm{D}_{2} \mathrm{O}$-exchangeable $\left.\mathrm{NH}\right), 13.11$ (s, 1H, $\mathrm{D}_{2} \mathrm{O}$-exchangeable $\left.\mathrm{NH}\right) ; \mathrm{MS} m / z(\%): 344\left(\mathrm{M}^{+}+1,4.8\right), 343$ $\left(\mathrm{M}^{+}, 13.5\right), 172$ (86.5), 156 (62.9), 143 (99.1), 65 (100). Anal. Calcd for $\mathrm{C}_{16} \mathrm{H}_{13} \mathrm{~N}_{3} \mathrm{O}_{4} \mathrm{~S}$ (343.35): C, 55.97; H, 3.82; N, 12.24; S, 9.34. Found: C, 55.90; H, 3.80; N, 12.20; S, 9.31\%. 


\subsubsection{2-Oxo- $N$-(4-sulfamoylphenyl)-2H-chromene-3-carboxamide (12)}

Method A: To a solution of $3(1.20 \mathrm{~g}, 5 \mathrm{mmol})$ in acetic acid $(30 \mathrm{~mL})$ containing $0.5 \mathrm{~g}$ of fused sodium acetate, salicyaldehyde $(0.61 \mathrm{~g}, 5 \mathrm{mmol})$ was added. The mixture was heated under reflux for $2 \mathrm{~h}$. After cooling, the formed product was collected by filtration and recrystallized from DMF to give $\mathbf{1 2}$.

Method B: The iminochromene derivatives 11 (0.86 g, $2.5 \mathrm{mmol})$ was dissolved in boiling dioxane $(40 \mathrm{~mL})$ and treated with $5 \mathrm{~mL} \mathrm{HCl}$. The reaction mixture was heated under reflux for $2 \mathrm{~h}$. Left to cool, the obtained product was filtered off, washed with cold water, and air-dried. Yield (86\%), mp $>300{ }^{\circ} \mathrm{C}$ (from DMF); IR (KBr) v $v_{\max }$ : 3362, 3258, $3108\left(\mathrm{NH}, \mathrm{NH}_{2}\right), 1698(\mathrm{C}=\mathrm{O}) \mathrm{cm}^{-1}$; ${ }^{1} \mathrm{H}-\mathrm{NMR}$ (DMSO- $\left.d_{6}\right)$ : $\delta 7.30$ (s, 2H, $\mathrm{D}_{2} \mathrm{O}$-exchangeable $\mathrm{NH}_{2}$ ), 7.57 (d, 2H, $\left.J=9 \mathrm{~Hz}, \mathrm{ArH}\right), 7.79-7.89$ (m, 4H, ArH), 8.03 (d, $2 \mathrm{H}, J=9 \mathrm{~Hz}, \mathrm{ArH}), 8.92(\mathrm{~s}, 1 \mathrm{H}, \mathrm{CH}), 10.88$ (s, $1 \mathrm{H}, \mathrm{D}_{2} \mathrm{O}$-exchangeable $\left.\mathrm{NH}\right) ;{ }^{13} \mathrm{C}-\mathrm{NMR}: \delta 114.9$, 118.7, 119.8, 124.3, 126.9, 130.2, 134.6, 139.1, 141.2, 142.1, 153.5, 155.4, 160.2, 161.2; MS m/z (\%): $345\left(\mathrm{M}^{+}+1,3.6\right), 344\left(\mathrm{M}^{+}, 10.1\right), 224$ (2.8), 173 (100), 118 (5.8), 101 (47.3), 90 (14.6), 80 (14.2), 76 (6.5), 64 (47.0). Anal. Calcd for $\mathrm{C}_{16} \mathrm{H}_{12} \mathrm{~N}_{2} \mathrm{O}_{5} \mathrm{~S}$ (344.34): C, 55.81; H, 3.51; N, 8.14; S, 9.31. Found: C, 55.76; H, 3.45; N, 8.10; S, 9.29\%.

\subsubsection{Coupling of $N$-[4-(Aminosulfonyl)phenyl]-2-cyanoacetamide (3) with the Appropriate} Diazonium Salt of Aromatic Amines

General procedure: To a cold solution of cyanoacetanilide $3(1.20 \mathrm{~g}, 5 \mathrm{mmol})$ in pyridine $(20 \mathrm{~mL})$, was added the appropriate diazonium salt of aromatic amine (4-methylaniline or 4-methoxyaniline or aniline or 4-chloroaniline or methyl 2-aminobenzoate) $(5 \mathrm{mmol})$ [prepared according to literature procedures] [32]. The addition was carried out portion wise with stirring at $0-5{ }^{\circ} \mathrm{C}$ over a period of $30 \mathrm{~min}$. After complete addition, the reaction mixture was stirred for a further $4 \mathrm{~h}$ then kept in an ice chest for $12 \mathrm{~h}$ and finally diluted with water. The precipitated solid was collected by filtration, washed with water, dried and finally recrystallized from the proper solvent to afford the corresponding coupling products 13a-e.

\subsubsection{2-Cyano-2-[2-(4-methylphenyl)hydrazinylidene]- $N$-(4-sulfamoylphenyl)ethanamide (13a)}

Yield (94\%), mp $288{ }^{\circ} \mathrm{C}$ (from dioxane); IR (KBr) $v_{\max }: 3325,3226,3186\left(\mathrm{NH}, \mathrm{NH}_{2}\right), 2214$ $(\mathrm{C} \equiv \mathrm{N}), 1664(\mathrm{C}=\mathrm{O}) \mathrm{cm}^{-1}$; ${ }^{1} \mathrm{H}-\mathrm{NMR}\left(\mathrm{DMSO}-d_{6}\right): \delta 2.30\left(\mathrm{~s}, 3 \mathrm{H}, \mathrm{CH}_{3}\right), 7.20\left(\mathrm{~s}, 2 \mathrm{H}, \mathrm{D}_{2} \mathrm{O}\right.$-exchangeable $\mathrm{NH}_{2}$ ), 7.22-7.64 (m, 4H, ArH), 7.81 (d, 2H, $\left.J=9 \mathrm{~Hz}, \mathrm{ArH}\right), 7.92$ (d, 2H, $\left.J=9 \mathrm{~Hz}, \mathrm{ArH}\right), 10.13$ (s, 1H, $\mathrm{D}_{2} \mathrm{O}$-exchangeable $\left.\mathrm{NH}\right), 11.93\left(\mathrm{~s}, 1 \mathrm{H}, \mathrm{D}_{2} \mathrm{O}\right.$-exchangeable $\left.\mathrm{NH}\right) ; \mathrm{MS} m / z(\%): 358\left(\mathrm{M}^{+}+1,8.7\right)$, 357 ( $\left.\mathrm{M}^{+}, 45.0\right), 198$ (6.4), 186 (23.8), 172 (87.8), 134 (10.9), 119 (19.9), 106 (47.9), 91 (100), 77 (52.1), 65 (38.3). Anal. Calcd for $\mathrm{C}_{16} \mathrm{H}_{15} \mathrm{~N}_{5} \mathrm{O}_{3} \mathrm{~S}$ (357.38): C, 53.77; H, 4.23; N, 19.60; S, 8.97. Found: C, 53.71; $\mathrm{H}, 4.20 ; \mathrm{N}, 19.55 ; \mathrm{S}, 8.87 \%$.

\subsubsection{2-Cyano-2-[2-(4-methoxyphenyl)hydrazinylidene]- $N$-(4-sulfamoylphenyl)ethanamide (13b)}

Yield (95\%), mp $274{ }^{\circ} \mathrm{C}$ (from dioxane); IR (KBr) v $v_{\max }$ : 3336, $3227\left(\mathrm{NH}, \mathrm{NH}_{2}\right), 2212(\mathrm{C} \equiv \mathrm{N})$, $1662(\mathrm{C}=\mathrm{O}) \mathrm{cm}^{-1}$; ${ }^{1} \mathrm{H}-\mathrm{NMR}\left(\mathrm{DMSO}-d_{6}\right): \delta 3.77\left(\mathrm{~s}, 3 \mathrm{H}, \mathrm{OCH}_{3}\right), 7.00(\mathrm{~d}, 2 \mathrm{H}, J=9 \mathrm{~Hz}, \mathrm{ArH}), 7.25(\mathrm{~s}, 2 \mathrm{H}$, $\mathrm{D}_{2} \mathrm{O}$-exchangeable $\left.\mathrm{NH}_{2}\right), 7.69(\mathrm{~d}, 2 \mathrm{H}, J=9 \mathrm{~Hz}, \mathrm{ArH}), 7.81(\mathrm{~d}, 2 \mathrm{H}, J=8.7 \mathrm{~Hz}, \mathrm{ArH}), 7.92(\mathrm{~d}, 2 \mathrm{H}$, $J=8.7 \mathrm{~Hz}, \mathrm{ArH}), 10.10$ (s, 1H, $\mathrm{D}_{2} \mathrm{O}$-exchangeable $\left.\mathrm{NH}\right), 11.95$ (s, $1 \mathrm{H}, \mathrm{D}_{2} \mathrm{O}$-exchangeable $\mathrm{NH}$ ); 
MS m/z (\%): $374\left(\mathrm{M}^{+}+1,8.7\right), 373\left(\mathrm{M}^{+}, 24.3\right), 270$ (5.8), 175 (21.4), 172 (39.8 ), 129 ( 22.3), 122 (100), 107 (38.8), 92 (38.8), 77 (47.6). Anal. Calcd for $\mathrm{C}_{16} \mathrm{H}_{15} \mathrm{~N}_{5} \mathrm{O}_{4} \mathrm{~S}$ (373.38): C, 51.47; H, 4.05; N, 18.76; S, 8.59. Found: C, 51.36; H, 4.00; N, 18.69; S, 8.52\%.

\subsubsection{2-Cyano-2-(2-phenylhydrazinylidene)- $N$-(4-sulfamoylphenyl)ethanamide (13c)}

Yield (92\%), mp $>300{ }^{\circ} \mathrm{C}$ (from dioxane); IR (KBr) $v_{\max }: 3367,3269,3240\left(\mathrm{NH}, \mathrm{NH}_{2}\right), 2218(\mathrm{C} \equiv \mathrm{N})$, $1680(\mathrm{C}=\mathrm{O}) \mathrm{cm}^{-1} ;{ }^{1} \mathrm{H}-\mathrm{NMR}$ (DMSO-d $\left.)\right): \delta 7.26\left(\mathrm{~s}, 2 \mathrm{H}, \mathrm{D}_{2} \mathrm{O}\right.$-exchangeable $\left.\mathrm{NH}_{2}\right), 7.38-7.79(\mathrm{~m}, 5 \mathrm{H}$, ArH), 7.82 (d, 2H, J=9 Hz, ArH), 7.93 (d, 2H, J=9 Hz, ArH), 10.17 (s, 1H, D $\mathrm{D}_{2}$-exchangeable NH), 11.98 (s, 1H, $\mathrm{D}_{2} \mathrm{O}$-exchangeable NH); MS m/z (\%): 343 ( $\left.\mathrm{M}^{+}, 36.8\right), 206$ (21.1), 198 (18.4), 172 (68.4), 145 (50.0), 118 (31.6), 108 (36.8), 91 (84.2), 80 (50.0), 77 (76.3), 60 (100). Anal. Calcd for $\mathrm{C}_{15} \mathrm{H}_{13} \mathrm{~N}_{5} \mathrm{O}_{3} \mathrm{~S}$ (343.36): C, 52.47; H, 3.82; N, 20.40; S, 9.34. Found: C, C, 52.45; H, 3.78; N, 20.38; $\mathrm{S}, 9.30 \%$.

\subsubsection{2-[2-(4-Chlorophenyl)hydrazinylidene]-2-cyano- $N$-(4-sulfamoylphenyl)ethanamide (13d)}

Yield (95\%), $\mathrm{mp}>300{ }^{\circ} \mathrm{C}$ (from dioxane); IR (KBr) $v_{\max }: 3360,3232,3190\left(\mathrm{NH}, \mathrm{NH}_{2}\right), 2216(\mathrm{C} \equiv \mathrm{N})$, $1668(\mathrm{C}=\mathrm{O}) \mathrm{cm}^{-1} ;{ }^{1} \mathrm{H}-\mathrm{NMR}\left(\mathrm{DMSO}-d_{6}\right): \delta 7.26\left(\mathrm{~s}, 2 \mathrm{H}, \mathrm{D}_{2} \mathrm{O}\right.$-exchangeable $\left.\mathrm{NH}_{2}\right), 7.47(\mathrm{~d}, 2 \mathrm{H}, J=9 \mathrm{~Hz})$, 7.74-7.82 (m, 4H, ArH), 7.92 (d, 2H, $J=9 \mathrm{~Hz}), 10.22$ (s, 1H, $\mathrm{D}_{2} \mathrm{O}$-exchangeable NH), 12.04 (s, 1H, $\mathrm{D}_{2} \mathrm{O}$-exchangeable $\left.\mathrm{NH}\right)$; $\mathrm{MS} m / z(\%): 379\left(\mathrm{M}^{+}+2,17.9\right), 378\left(\mathrm{M}^{+}+1,12.5\right), 377\left(\mathrm{M}^{+}, 39.3\right)$, 296 (26.8), 238 (12.5 ), 206 (41.1), 182 (32.1), 172 (96.4), 156 (51.8), 125 (57.1), 111 (53.6), 106 (30.4), 90 (69.6), 77 (21.4), 64 (100). Anal. Calcd for $\mathrm{C}_{15} \mathrm{H}_{12} \mathrm{ClN}_{5} \mathrm{O}_{3} \mathrm{~S}$ (377.80): C, 47.69; H, 3.20; Cl, 9.38; N, 18.54; S, 8.49. Found: C, 47.61; H, 3.15; Cl, 9.27; N, 18.49; S, 8.43\%.

3.2.18. Methyl 4-[2-(1-Cyano-2-oxo-2-\{(4-sulfamoylphenyl)amino\}ethylidene)hydrazinyl]benzoate (13e)

Yield (95\%), $\mathrm{mp}>300^{\circ} \mathrm{C}$ (from dioxane); IR (KBr) $v_{\max }: 3340,3231,3148\left(\mathrm{NH}, \mathrm{NH}_{2}\right) 2211(\mathrm{C} \equiv \mathrm{N})$, $1687(\mathrm{C}=\mathrm{O}) \mathrm{cm}^{-1}$; ${ }^{1} \mathrm{H}-\mathrm{NMR}\left(\mathrm{DMSO}-d_{6}\right): \delta 3.94\left(\mathrm{~s}, 3 \mathrm{H}, \mathrm{CH}_{3}\right), 7.24\left(\mathrm{~s}, 2 \mathrm{H}, \mathrm{D}_{2} \mathrm{O}\right.$-exchangeable $\mathrm{NH}_{2}$ ), 7.31-7.94 (m, 4H, ArH), 8.03 (d, 2H, $J=9 \mathrm{~Hz}), 8.32$ (d, 2H, $J=9 \mathrm{~Hz}), 10.43$ (s, 1H, $\mathrm{D}_{2} \mathrm{O}$-exchangeable $\mathrm{NH}), 12.49$ (s, 1H, $\mathrm{D}_{2} \mathrm{O}$-exchangeable $\left.\mathrm{NH}\right)$; $\mathrm{MS} m / z(\%)$ : $402\left(\mathrm{M}^{+}+1,3.0\right), 401\left(\mathrm{M}^{+}, 12.8\right), 170$ (11.7), 133 (100), 90 (34.1), 92 (20.2), 77 (21.2), 65 (12.7). Anal. Calcd for $\mathrm{C}_{17} \mathrm{H}_{15} \mathrm{~N}_{5} \mathrm{O}_{5} \mathrm{~S}$ (401.39): C, 50.87; H, 3.77; N, 17.45; S, 7.99. Found: C, 50.81; H, 3.65; N, 17.40; S, 7.92\%.

\subsubsection{Synthesis of Aminopyrazoles 14a,b}

To a solution of the compound 13a,b $(5 \mathrm{mmol})$ in dioxane $(20 \mathrm{~mL})$, hydrazine hydrate $(80 \%$, $1.0 \mathrm{~mL}, 5 \mathrm{mmol}$ ) was added and the reaction mixture was refluxed for $6 \mathrm{~h}$ and allowed to cool. The solid product obtained was filtered, washed with $\mathrm{EtOH}$ and dried. Recrystallization from dioxane afforded 14a,b.

3.2.20. 4-(\{5-Amino-4-[(4-methylphenyl)diazenyl]-1H-pyrazol-3-yl $\}$ amino)benzenesulfonamide (14a)

Yield (40\%), mp $240{ }^{\circ} \mathrm{C}$ (from dioxane); IR (KBr) $v_{\max }: 3459,3367,3345,3208\left(\mathrm{NH}, \mathrm{NH}_{2}\right), 2920$, 2859 (aliphaticCH) cm ${ }^{-1} ;{ }^{1} \mathrm{H}-\mathrm{NMR}$ (DMSO- $\left.d_{6}\right): \delta 2.29\left(\mathrm{~s}, 3 \mathrm{H}, \mathrm{CH}_{3}\right), 5.18\left(\mathrm{~s}, 2 \mathrm{H}, \mathrm{D}_{2} \mathrm{O}\right.$-exchangeable $\left.\mathrm{NH}_{2}\right), 7.23(\mathrm{~d}, 2 \mathrm{H}, J=9 \mathrm{~Hz}), 7.42(\mathrm{~d}, 2 \mathrm{H}, J=9 \mathrm{~Hz}), 7.49$ (s, 2H, $\mathrm{D}_{2} \mathrm{O}$-exchangeable $\left.\mathrm{NH}_{2}\right), 7.75-7.90$ 
(m, 4H, ArH), 9.86 (s, 1H, $\mathrm{D}_{2} \mathrm{O}$-exchangeable $\left.\mathrm{NH}\right), 13.65$ (s, 1H, $\mathrm{D}_{2} \mathrm{O}$-exchangeable $\left.\mathrm{NH}\right) ;{ }^{13} \mathrm{C}-\mathrm{NMR}$ : $\delta 20.5,99.4,117.0,119.5,126.4,129.5,132.9,137.9,141.8,145.1,151.5,165.7 ; \mathrm{MS} m / z(\%)$ : $372\left(\mathrm{M}^{+}+1\right.$, 14.6), 356 (20.8), 217 ( 31.3 ), 201 (16.7), 156 ( 31.3 ), 126 ( 22.9), 123 (58.3), 107 (50.0), 106 (100), 90 (50.0), 77 (54.2). Anal. Calcd for $\mathrm{C}_{16} \mathrm{H}_{17} \mathrm{~N}_{7} \mathrm{O}_{2} \mathrm{~S}$ (371.41): C, 51.74; H, 4.61; N, 26.40; S, 8.63. Found: C, 51.70; H, 4.58; N, 26.20; S, 8.59\%.

3.2.21. 4-(\{5-Amino-4-[(4-methoxyphenyl)diazenyl]-1H-pyrazol-3-yl $\}$ amino $)$ benzenesulfonamide (14b)

Yield (72\%), mp $200{ }^{\circ} \mathrm{C}$ (from dioxane); IR (KBr) $v_{\max }: 3436,3351,3259\left(\mathrm{NH}, \mathrm{NH}_{2}\right.$ ), $1658(\mathrm{C}=\mathrm{O}) \mathrm{cm}^{-1} ;{ }^{1} \mathrm{H}-\mathrm{NMR}\left(\mathrm{DMSO}-d_{6}\right): \delta 3.77\left(\mathrm{~s}, 3 \mathrm{H}, \mathrm{OCH}_{3}\right), 5.03\left(\mathrm{~s}, 2 \mathrm{H}, \mathrm{D}_{2} \mathrm{O}\right.$-exchangeable $\left.\mathrm{NH}_{2}\right)$, $7.00(\mathrm{~d}, 2 \mathrm{H}, J=9 \mathrm{~Hz}), 7.21\left(\mathrm{~s}, 2 \mathrm{H}, \mathrm{D}_{2} \mathrm{O}\right.$-exchangeable $\left.\mathrm{NH}_{2}\right), 7.49(\mathrm{~d}, 2 \mathrm{H}, J=9 \mathrm{~Hz}), 7.77-7.81(\mathrm{~m}, 4 \mathrm{H}$, $\operatorname{ArH}), 9.70\left(\mathrm{~s}, 1 \mathrm{H}, \mathrm{D}_{2} \mathrm{O}\right.$-exchangeable $\left.\mathrm{NH}\right), 13.20$ (s, 1H, $\mathrm{D}_{2} \mathrm{O}$-exchangeable $\left.\mathrm{NH}\right) ; \mathrm{MS} \mathrm{m} / z$ (\%): $389\left(\mathrm{M}^{+}+2,35.4\right), 388\left(\mathrm{M}^{+}+1,40.2\right), 387\left(\mathrm{M}^{+}, 38.4\right), 250$ (31.7), 216 (40.2), 196 (53.0), 171 (45.1), 155 (31.7), 122 (33.5), 108 (34.8), 95 (40.2), 69 (100). Anal. Calcd for $\mathrm{C}_{16} \mathrm{H}_{17} \mathrm{~N}_{7} \mathrm{O}_{3} \mathrm{~S}$ (387.41): C, 49.60; H, 4.42; N, 25.31; S, 8.28. Found: C, 49.58; H, 4.40; N, 25.30; S, 8.20\%.

3.2.22. 4-Amino-3-phenyl- $N$-(4-sulfamoylphenyl)-2-thioxo-2,3-dihydro-1,3-thiazole-5-carboxamide (15)

To a solution of cyanoacetamide $3(1.20 \mathrm{~g}, 5 \mathrm{mmol})$ in DMF containing triethylamine $(1 \mathrm{~mL})$, elemental sulfur $(0.16 \mathrm{~g}, 5 \mathrm{mmol})$ and phenyl isothiocyanate $(0.68 \mathrm{~mL}, 5 \mathrm{mmol})$ were added. The reaction mixture was heated at $60{ }^{\circ} \mathrm{C}$ for $2 \mathrm{~h}$ with continous stirring and then poured into a beaker containing an ice-water mixture with few drops of $\mathrm{HCl}$. The solid product so formed was filtered off, washed with EtOH and dried. Recrystallization from dioxane afforded compound 15. Yield (60\%), $\mathrm{mp}>300^{\circ} \mathrm{C}$ (from DMF); IR (KBr) $v_{\max }$ : 3397, $3210\left(\mathrm{NH}, \mathrm{NH}_{2}\right), 1671(\mathrm{C}=\mathrm{O}), 1335,1217(\mathrm{C}=\mathrm{S}) \mathrm{cm}^{-1}$; ${ }^{1} \mathrm{H}-\mathrm{NMR}\left(\mathrm{DMSO}-d_{6}\right): \delta 3.42\left(\mathrm{~s}, 2 \mathrm{H}, \mathrm{D}_{2} \mathrm{O}\right.$-exchangeable $\mathrm{NH}_{2}$ ), $7.19\left(\mathrm{~s}, 2 \mathrm{H}, \mathrm{D}_{2} \mathrm{O}\right.$-exchangeable $\mathrm{NH}_{2}$ ), $7.50(\mathrm{~d}, 2 \mathrm{H}, J=9 \mathrm{~Hz}), 7.55-7.88(\mathrm{~m}, 5 \mathrm{H}, \mathrm{ArH}), 7.96(\mathrm{~d}, 2 \mathrm{H}, J=9 \mathrm{~Hz}), 8.45\left(\mathrm{~s}, 1 \mathrm{H}, \mathrm{D}_{2} \mathrm{O}\right.$-exchangeable $\mathrm{NH})$; MS $m / z(\%) 406\left(\mathrm{M}^{+}, 0.9\right), 402$ (5.7), 172 (14.3), 156 (8.6), 129 (11.4), 114 (11.4), 109 (17.1), 82 (32.9), 76 (44.3), 63 (100). Anal. Calcd for $\mathrm{C}_{16} \mathrm{H}_{14} \mathrm{~N}_{4} \mathrm{O}_{3} \mathrm{~S}_{3}$ (406.50): C, 47.27; H, 3.47; N, 13.78; S, 23.66. Found: C, 47.21; H, 3.44; N, 13.71; S, 23.59\%.

\subsubsection{3,5-Diamino-4-cyano- $N$-(4-sulfamoylphenyl)thiophene-2-carboxamide (16)}

To a solution of compound $3(1.20 \mathrm{~g}, 5 \mathrm{mmol})$ in dioxane $(25 \mathrm{~mL})$ containing triethylamine $(1.00 \mathrm{~mL})$, malononitrile $(0.33 \mathrm{~g}, 5 \mathrm{mmol})$ was added followed by the addition of an equimolar amount of elemental sulfur $(0.16 \mathrm{~g}, 5 \mathrm{mmol})$. The reaction mixture was heated under reflux for $5 \mathrm{~h}$, then cooled and neutralized by pouring onto ice/water mixture containing few drops of hydrochloric acid. The solid product formed was collected by filtration and crystallized from dioxane. Yield $(86 \%), \mathrm{mp}>300{ }^{\circ} \mathrm{C}$ (from dioxane); IR (KBr) $v_{\max }$ : 3743, $3316\left(\mathrm{NH}, \mathrm{NH}_{2}\right), 2209(\mathrm{C} \equiv \mathrm{N}), 1635(\mathrm{C}=\mathrm{O}) \mathrm{cm}^{-1} ;{ }^{1} \mathrm{H}-\mathrm{NMR}$ (DMSO- $d_{6}$ ): $\delta 3.45$ (s, 4H, $\mathrm{D}_{2} \mathrm{O}$-exchangeable $2 \mathrm{NH}_{2}$ ), $7.26\left(\mathrm{~s}, 2 \mathrm{H}, \mathrm{D}_{2} \mathrm{O}\right.$-exchangeable $\mathrm{NH}_{2}$ ), 7.50-7.92 (m, 4H, ArH), 11.36 (s, 1H, $\mathrm{D}_{2} \mathrm{O}$-exchangeable $\left.\mathrm{NH}\right)$; $\mathrm{MS} m / z(\%): 338\left(\mathrm{M}^{+}+1,44.9\right), 337\left(\mathrm{M}^{+}, 47.2\right)$, 295 (47.2), 278 (45.7), 181 (49.6), 156 (53.5), 150 (51.9), 122 (51.2), 105 (64.6), 80 (48.8), 58 (100.0). Anal. Calcd for $\mathrm{C}_{12} \mathrm{H}_{11} \mathrm{~N}_{5} \mathrm{O}_{3} \mathrm{~S}$ (337.37): C, 42.72; H, 3.29; N, 20.76; S, 19.01. Found: C, 42.68; H, 3.21; N, 20.66; S, 18.96\%. 


\subsubsection{Synthesis of $\mathbf{1 8}, \mathbf{1 9}$ and $\mathbf{2 0}$}

Compound 3 (1.20 g, $5 \mathrm{mmol})$ was added to a stirred solution of potassium hydroxide $(0.26 \mathrm{~g}$, $5 \mathrm{mmol})$ in DMF $(20 \mathrm{~mL})$. After stirring for $30 \mathrm{~min}$, phenyl isothiocyanate $(0.68 \mathrm{~g}, 5 \mathrm{mmol})$ was added to the resulting mixture. Stirring was continued for $6 \mathrm{~h}$, and then chloroacetone or 3-(2-bromoacetyl)$2 \mathrm{H}$-chromen-2-one or methyl iodide, $(5 \mathrm{mmol}$ ) was added portion wise over a period of $30 \mathrm{~min}$. After the addition was complete, the reaction mixture was stirred for an additional $12 \mathrm{~h}$, during which the reactant dissolved and a yellow product precipitated. The solid product was filtered off, washed with EtOH and dried. Recrystallization from proper solvent afforded 18, 19 and 20.

3.2.25. $N$-[4-(Aminosulfonyl)phenyl]-2-cyano-2-(4-methyl-3-phenyl-1,3-thiazol-2(3H)-ylidene)acetamide (18)

Yield (95\%), mp $272{ }^{\circ} \mathrm{C}$ (from Dioxane) (lit. mp 260-262 ${ }^{\circ} \mathrm{C}$ ) [31]; IR (KBr) $v_{\max }: 3294,3196,3107$ $\left(\mathrm{NH}, \mathrm{NH}_{2}\right), 2178(\mathrm{C} \equiv \mathrm{N}), 1605(\mathrm{C}=\mathrm{O}) \mathrm{cm}^{-1} ;{ }^{1} \mathrm{H}-\mathrm{NMR}\left(\mathrm{DMSO}-d_{6}\right): \delta 1.86\left(\mathrm{~s}, 3 \mathrm{H}, \mathrm{CH}_{3}\right), 6.98(\mathrm{~s}, 1 \mathrm{H}$, thiazole-CH), 7.17 (s, 2H, $\mathrm{D}_{2} \mathrm{O}$-exchangeable $\left.\mathrm{NH}_{2}\right), 7.49$ (d, 2H, $\left.J=9 \mathrm{~Hz}\right), 7.57$ (d, 2H, $\left.J=9 \mathrm{~Hz}\right)$, 7.60-7.70 (m, 5H, ArH), $9.02\left(\mathrm{~s}, 1 \mathrm{H}, \mathrm{D}_{2} \mathrm{O}\right.$-exchangeable $\left.\mathrm{NH}\right) ;{ }^{13} \mathrm{C}-\mathrm{NMR}$ (DMSO- $d_{6}$ ): $\delta 14.2,66.3$, 106.9, 115.9, 119.3, 126.2, 128.9, 129.6, 130.6, 136.6, 137.5, 138.3, 142.4, 165.1, 166.5; MS m/z (\%): $412\left(\mathrm{M}^{+}, 10.1\right), 332$ (2.1), 241 (100), 214 (35.3), 198 (19.2), 172 (6.9), 118 (19.9), 90 (38.3), 77 (43.2). Anal. Calcd for $\mathrm{C}_{19} \mathrm{H}_{16} \mathrm{~N}_{4} \mathrm{O}_{3} \mathrm{~S}_{2}$ (412.48): C, 55.32; H, 3.91; N, 13.58; S, 15.55. Found: C, 55.30; H, 3.88; N, 13.51; S, $15.50 \%$.

3.2.26. 2-Cyano-2-[4-(2-oxo-2H-chromen-4-yl]-3-phenyl-1,3-thiazol-2(3H)-ylidene)- $N$-(4-sulfamoylphenyl)ethanamide (19)

Yield (55\%), mp $280{ }^{\circ} \mathrm{C}$ (from Dioxane); IR (KBr) $v_{\max }: 3367,3310$ and $3235\left(\mathrm{NH}, \mathrm{NH}_{2}\right), 3060$ (aromatic CH), $1713(\mathrm{C}=\mathrm{O}), 1635(\mathrm{C}=\mathrm{O}) \mathrm{cm}^{-1}$; ${ }^{1} \mathrm{H}-\mathrm{NMR}\left(\mathrm{DMSO}-d_{6}\right): \delta 1.01\left(\mathrm{~s}, 3 \mathrm{H}, J=7.2 \mathrm{~Hz}, \mathrm{CH}_{3}\right)$, 4.04 (q, 2H, $\left.J=7.2 \mathrm{~Hz}, \mathrm{CH}_{2}\right), 7.08-7.13$ (m, 5H, ArH), 7.21 (d, 2H, $\left.J=9 \mathrm{~Hz}\right), 7.39-7.44$ (m, 7H, ArH and $\left.\mathrm{NH}_{2}\right), 7.63(\mathrm{~d}, 2 \mathrm{H}, J=9 \mathrm{~Hz}), 9.54\left(\mathrm{~s}, 1 \mathrm{H}, \mathrm{D}_{2} \mathrm{O}\right.$-exchangeable $\left.\mathrm{NH}\right), 9.73\left(\mathrm{~s}, 1 \mathrm{H}, \mathrm{D}_{2} \mathrm{O}\right.$-exchangeable $\mathrm{NH}) ;{ }^{13} \mathrm{C}-\mathrm{NMR}: \delta 70.9,95.3,115.4,115.8,118.0,119.6,120.9,124.8,125.8,126.3,128.4,128.8,129.3$, $129.5,132.7,137.0,138.0,142.1,143.0,153.2,158.4,164.7,171.3 ; \mathrm{MS} m / z(\%): 521\left(\mathrm{M}^{+}, 26.3\right)$, 322 (15.8), 218 (100.0), 199 (15.8), 77 (86.0). Anal. Calcd for $\mathrm{C}_{27} \mathrm{H}_{18} \mathrm{~N}_{4} \mathrm{O}_{5} \mathrm{~S}_{2}$ (542.58): C, 59.77; H, 3.34; N, 10.33; S, 11.82. Found: C, 59.75; H, 3.30; N, 10.31; S, 11.79\%.

3.2.27. 2-Cyano-3-(methylsulfanyl)-3-(phenylamino)- $N$-(4-sulfamoylphenyl)prop-2-enamide (20)

Yield (95\%), mp $204{ }^{\circ} \mathrm{C}$ (from EtOH) (lit. mp 220-222 ${ }^{\circ} \mathrm{C}$ ) [33]; IR (KBr) v $\max : 3410,3339,3243$, $3119\left(\mathrm{NH}, \mathrm{NH}_{2}\right), 2194(\mathrm{C} \equiv \mathrm{N}), 1628(\mathrm{C}=\mathrm{O}) \mathrm{cm}^{-1} ;{ }^{1} \mathrm{H}-\mathrm{NMR}\left(\mathrm{DMSO}-d_{6}\right): \delta 2.49\left(\mathrm{~s}, 3 \mathrm{H}, \mathrm{CH}_{3}\right), 7.18(\mathrm{~s}, 2 \mathrm{H}$, $\mathrm{D}_{2} \mathrm{O}$-exchangeable $\left.\mathrm{NH}_{2}\right), 7.31-7.41(\mathrm{~m}, 5 \mathrm{H}, \mathrm{ArH}), 7.67(\mathrm{~d}, 2 \mathrm{H}, J=9 \mathrm{~Hz}), 7.79(\mathrm{~d}, 2 \mathrm{H}, J=9 \mathrm{~Hz}), 9.65$ (s, $1 \mathrm{H}, \mathrm{D}_{2} \mathrm{O}$-exchangeable $\left.\mathrm{NH}\right), 9.91\left(\mathrm{~s}, 1 \mathrm{H}, \mathrm{D}_{2} \mathrm{O}\right.$-exchangeable $\left.\mathrm{NH}\right) ; \mathrm{MS} m / z(\%): 389\left(\mathrm{M}^{+}+1,63.0\right)$, $388\left(\mathrm{M}^{+}, 10.9\right), 341$ (58.7), 294 (100), 249 (56.5), 223 (67.4), 208 (68.5), 197 (58.7), 185 (25.0), 182 (59.8), 140 (76.1), 92 (17.4). Anal. Calcd for $\mathrm{C}_{17} \mathrm{H}_{16} \mathrm{~N}_{4} \mathrm{O}_{3} \mathrm{~S}_{2}$ (388.46): C, 52.56; H, 4.15; N, 14.42; S, 16.51. Found: C, 52.50; H, 4.12; N, 14.45; S, 16.48\%. 


\subsubsection{2-Cyano-2-(1,3-dithian-2-ylidene)- $N$-(4-sulfamoylphenyl)acetamide (22)}

To a stirred suspension of finely powdered potassium hydroxide $(0.26 \mathrm{~g}, 5 \mathrm{mmole})$ in dry DMF (20 mL) cyanoacetamide $3(1.20 \mathrm{~g}, 5 \mathrm{mmole})$ was added, the resulted mixture was cooled at $10{ }^{\circ} \mathrm{C}$ in an ice bath, then carbon disulfide ( $5 \mathrm{mmol}$ ) was added slowly over the course of $10 \mathrm{~min}$. After addition was complete, stirring of the reaction mixture was continued for additional $2 \mathrm{~h}$. Then dibromopropane (5 mmol) was added to the mixture while cooling $\left(\sim 15^{\circ} \mathrm{C}\right)$ and stirring for $1 \mathrm{~h}$. The mixture was then poured into crushed ice and the resulting precipitate was filtrated off, dried and crystallized from the proper solvent to give 22. Yield (95\%), mp $278{ }^{\circ} \mathrm{C}$ (from Dioxane); IR (KBr) $v_{\max }: 3314,3241,3107$ $\left(\mathrm{NH}, \mathrm{NH}_{2}\right), 2209(\mathrm{C} \equiv \mathrm{N}), 1653(\mathrm{C}=\mathrm{O}) \mathrm{cm}^{-1} ;{ }^{1} \mathrm{H}-\mathrm{NMR}$ (DMSO-d $): \delta 2.18\left(\mathrm{~m}, 2 \mathrm{H}, J=6.80 \mathrm{~Hz}, \mathrm{CH}_{2}\right)$, $3.04\left(\mathrm{t}, 2 \mathrm{H}, J=6.6 \mathrm{~Hz}, \mathrm{CH}_{2}\right), 3.19$ (t, 2H, $\left.J=6.6 \mathrm{~Hz}, \mathrm{CH}_{2}\right), 7.25$ (s, 2H, $\mathrm{D}_{2} \mathrm{O}$-exchangeable $\mathrm{NH}_{2}$ ), 7.75 (m, 4H, ArH), 10.29 (s, 1H, $\mathrm{D}_{2} \mathrm{O}$-exchangeable $\left.\mathrm{NH}\right) ;{ }^{13} \mathrm{C}-\mathrm{NMR}: \delta 22.5,29.2,98.5,115.6,119.8$, 126.5, 139.0, 141.3, 159.8, 177.3; MS m/z (\%): $356\left(\mathrm{M}^{+}+1,2.9\right), 355\left(\mathrm{M}^{+}, 14.8\right), 237$ (0.2), 183 (100), 129 (2.3), 118 (1.2), 110 (27.6), 80 (18.1). Anal. Calcd for $\mathrm{C}_{13} \mathrm{H}_{13} \mathrm{~N}_{3} \mathrm{O}_{3} \mathrm{~S}_{3}$ (355.45): C, 43.93; H, 3.69; N, 11.82; S, 27.06. Found: C, 43.85; H, 3.57; N, 11.78; S, 27.00\%.

\subsection{Antimicrobial Evaluation}

The antibacterial and antifungal activity assays were carried out in the Medical Mycology Laboratory of the Regional Center for Mycology and Biotechnology of Al-Azhar University, Cairo, Egypt using the diffusion plate method [26-28] as follows: a bottomless cylinder containing a measured quantity $(1 \mathrm{~mL}, 5 \mathrm{mg} / \mathrm{mL})$ of the sample is placed on a plate $(9 \mathrm{~cm}$ diameter) containing a solid bacterial medium (nutrient agar broth) or fungal medium, which has been heavily seeded with a spore suspension of the test organism. After incubation ( $24 \mathrm{~h}$ for bacteria and 5 days for fungi), the diameter of the clear zone of inhibition surrounding the sample is taken as measure of the inhibitory power of the sample against the particular test organism. The solvent used was DMSO and the concentration of the sample used is $100 \mu \mathrm{g} / \mathrm{mL}$. The results of antimicrobial activity are summarized in Table 1 .

\section{Conclusions}

In conclusion, the reactivity of $N$-[4-(aminosulfonyl)phenyl]-2-cyanoacetamide (3) was investigated as a versatile and readily accessible building block for the synthesis of new heterocycles incorporating a sulfamoyl moiety of biological and pharmaceutical importance.

\section{Acknowledgments}

The authors would like to thank the Chemistry Department, Faculty of Science, Cairo University for their financial support to facilitate the publication of this study.

\section{Conflicts of Interest}

The authors declare no conflict of interest. 


\section{References}

1. El-Gaby, M.S.A.; Atalla, A.A.; Gaber, A.M.; Abd Al-Wahab, K.A. Studies on aminopyrazoles: Antibacterial activity of some novel pyrazolo[1,5-a]pyrimidines containing sulfonamido moieties. Il Farm. 2000, 55, 596-602.

2. El-Gaby, M.S.A.; Taha, N.M.; Micky, J.A.; El-Sharief, M.A.M.Sh. Preparation of some novel 3, 5-diaminopyrazole, pyrazolo $[1,5-a][1,3,5]$ triazine and pyrazolo $[1,5-a]$ pyrimidine derivatives containing sulfonamido moieties as antimicrobial agents. Acta Chim. Slov. 2002, 49, 159-171.

3. El-Gaby, M.S.A.; Gaber, A.M.; Atalla, A.A.; Abd Al-Wahab, K.A. Novel synthesis and antifungal activity of pyrrole and pyrrolo[2,3- $d]$ pyrimidine derivatives containing sulfonamido moieties. Il Farm. 2002, 57, 613-617.

4. Maren, T.H. Relations between structure and biological activity of sulfonamides. Annu. Rev. Pharmacol. Toxicol. 1976, 16, 309-327.

5. Alafeefy, A.M.; Isik, S.; Abdel-Aziz, H.A.; Ashour, A.E.; Vullo, D.; Al-Jaber, N.A.; Supuran, C.T. Carbonic anhydrase inhibitors: Benzenesulfonamides incorporating cyanoacrylamide moieties are low nanomolar/subnanomolar inhibitors of the tumor-associated isoforms IX and XII. Bioorg. Med. Chem. 2013, 21, 1396-1403.

6. Roifman, C.M.; Aviv, G.; Alexander, L. Preparation of $N$-benzyl-3-aryl-2-cyanoacrylamides activity for treatment of neoplastic disorders. PCT. Int. Appl. WO Patent 2000, 0055, 128; Chem. Abstr. 133, 237695h.

7. Fadda, A.A.; Mukhtar, M.M.; Refat, H.M. Utility of activated nitriles in the synthesis of some new heterocyclic compounds. Am. J. Org. Chem. 2012, 2, 32-40.

8. Labro, M.T. Immunomodulation by antibacterial agents: Is it clinically relevant. Drugs 1993, 45, 319-328.

9. Azab, M.E.; Youssef, M.M.; El-Bordany, E.A. Synthesis and antibacterial evaluation of novel heterocyclic compounds containing a sulfonamido moiety. Molecules 2013, 18, 832-844.

10. Abu-Hashem, A.A.; Gouda, M.A.; Badria, F.A. Synthesis of some new pyrimido [2',1':2,3] thiazolo[4,5-b]quinoxaline derivatives as anti-inflammatory and analgesic agents. Eur. J. Med. Chem. 2010, 45, 1976-1981.

11. Gouda, M.A.; Berghot, M.A.; Abd El-Ghani, G.E.; Khalil, A.M. Synthesis and antimicrobial activities of some new thiazole and pyrazole derivatives based on 4,5,6,7-tetrahydrobenzo thiophene moiety. Eur. J. Med. Chem. 2010, 45, 1338-1345.

12. Khalil, A.M.; Berghot, M.A.; Gouda, M.A. Synthesis and antibacterial activity of some new thiazole and thiophene derivatives. Eur. J. Med. Chem. 2009a, 44, 4434-4440.

13. Khalil, A.M.; Berghot, M.A.; Gouda, M.A.; Abd El-Ghani, G.E. Synthesis and antimicrobial evaluation of some new thiophene derivatives. Synth. Commun. 2010a, 40, 1658-1669.

14. Khalil, A.M.; Berghot, M.A.; Gouda, M.A.; Shoeib, A.I. Synthesis and antimicrobial of certain new thiazolidinone, thiazoline, and thiophene derivatives. Phosphorus Sulfur Silicon 2010b, 185, 1455-1462.

15. Wilby, M.J.; Hutchinson, P.J. The pharmacology of chlormethiazole: A potential neuroprotective agent? CNS Drug Rev. 2004, 10, 281-294. 
16. Harnett, J.J.; Roubert, V.; Dolo, C.; Charnet, C.; Spinnewyn, B.; Cornet, S.; Rolland, A.; Marin, J.G.; Bigg, D.; Chabrier, P.E. Phenolic thiazoles as novel orally-active neuroprotective agents. Bioorg. Med. Chem. Lett. 2004, 14, 157-160.

17. Anderson, W.K.; Dean, D.C.; Endo, T. Synthesis, chemistry, and antineoplastic activity of $\alpha$-halopyridinium salts: Potential pyridone prodrugs of acylated vinylogous carbinolamine tumor inhibitors. J. Med. Chem. 1990, 33, 1667-1675.

18. Li, Q.; Mitscher, L.A.; Shen, L.L. The 2-pyridone antibacterial agents: Bacterial topoisomerase inhibitors. Med. Res. Rev. 2000, 20, 231-293.

19. Dragovich, P.S.; Prins, T.J.; Zhou, R.; Brown, E.L.; Maldonado, F.C.; Fuhrman, S.A.; Zalman, L.S.; Tuntland, T.; Lee, C.A.; Patick, A.K.; et al. Structure-based design, synthesis, and biological evaluation of irreversible human rhinovirus $3 \mathrm{C}$ protease inhibitors. 6: Structure-activity studies of orally bioavailable, 2-pyridone-containing peptidomimetics. J. Med. Chem. 2002, 45, 1607-1623.

20. Dragovich, P.S.; Prins, T.J.; Zhou, R.; Johnson, T.O.; Brown, E.L.; Maldonado, F.C.; Fuhrman, S.A.; Zalman, L.S.; Patick, A.K.; Matthews, D.A.; et al. Structure-based design, synthesis, and biological evaluation of irreversible human rhinovirus 3C protease inhibitors. Part 7: Structure-activity studies of bicyclic 2-pyridone-containing peptidomimetics. Bioorg. Med. Chem. Lett. 2002, 12, 733-738.

21. Darwish, E.S.; Kheder, N.A.; Farag, A.M. Synthesis and antimicrobial evaluation of some new pyridine based heterocycles. Heterocycles 2010, 81, 2247-2256.

22. Darwish, E.S.; Mahmoud, F.F.; Hussein, A.M.; Altalbawy, F.M. $\beta$-Oxoanilides in heterocyclic: Synthesis of som new pyridazine and polyfunctionally substituted heterocyclic compounds. Arab. J. Chem. 2013, in press.

23. Ammar, Y.A.; Saleh, N.M.; Micky, J.A.; Abas, H.S.; El-Gaby, M.S.A. Activated nitriles in heterocyclic chemistry: Facile synthesis and antimicrobial activity of some pyrimidine, pyrazolopyrimidine and pyrazolotriazinederivatives containing sulfonamido moiety. Indian J. Chem. 2004, 43B, 2203-2211.

24. El-Bayouki, K.A.M.; Basyouni, W.M.; Aly, Y.A.M.M.; Abbas, S.Y. Novel 4(3H)-quinazolinones containing biologically active thiazole, pyridinone and chromene of expected antitumor and antifungal activities. Eur. J. Chem. 2011, 2, 455-462.

25. Gorobets, Y.N.; Yousefi, B.H.; Belaj, F.; Kappe, C.O. Rapid microwave-assisted solution phase synthesis of substituted 2-pyridone libraries. Tetrahedron 2004, 60, 8633-8644.

26. Esmail, R.; Kurzer, F. Heterocyclic compounds from urea derivatives. Part XXIII. Thiobenzoylated thiocarbonohydrazides and their cyclization. J. Chem. Soc. 1975, 18, 1787-1791.

27. Muanz, D.N.; Kim, B.W.; Euler, K.L.; Williams, L. Antibacterial and antifungal activities of nine medicinal plants from Zaire. Int. J. Pharmacogn. 1994, 32, 337-345.

28. Harborne, J.B.; Williams, C.A. A survey of antifungal compounds from higher plants, 1982-1993. Phytochemistry 1994, 37, 19-42.

29. Koelsch, C.F. Bromination of acetocoumarin. J. Am. Chem. Soc. 1950, 72, 2993-2995.

30. Czerney, P.; Hartman, H. 3- $\alpha$-Bromoacetyl-coumarines as synthones for heterocyclic substituted coumarines. J. Prakt. Chem. 1983, 325, 551-560. 
31. Ammar, Y.A.; Aly, M.M.; Al-Sehem, A.G.; Mohamed, Y.A.; Salem, M.A.; El-Gaby, M.S.A. Cyanoacetanilides intermediates in heterocyclic synthesis. Part 4: Preparation of some hitherto unknown thiazolidine and bisthiazolidine derivatives. Phosphorus Sulfur Silicon 2008, 183, 1710-1721.

32. Butler, R.N. The diazotization of heterocyclic primary amines. Chem. Rev. 1975, 75, 241-257.

33. Ammar, Y.A.; Aly, M.M.; Al-Sehemi, A.G.; Salem, M.A.; El-Gaby, M.S.A. Cyanoacetanilides intermediates in heterocyclic synthesis. Part 5: Preparation of hitherto unknown 5-aminopyrazole and pyrazolo[1,5-a]pyrimidine derivatives containing sulfamoyl moiety. J. Chin. Chem. Soc. 2009, 56, 1064-1071.

(C) 2014 by the authors; licensee MDPI, Basel, Switzerland. This article is an open access article distributed under the terms and conditions of the Creative Commons Attribution license (http://creativecommons.org/licenses/by/3.0/). 